\title{
LA OCUPACIÓN HUMANA ANTIGUA DE LOS CALLEJONES SIN SALIDA DE LOS ANDES DE PATAGONIA: MIDIENDO LA INTENSIDAD DE USO DEL ESPACIO EN LOS MÁRGENES DEL CAMPO DE HIELO NORTE (AISÉN, CHILE) ${ }^{1}$
}

\author{
ANCIENT HUMAN OCCUPATION OF THE PATAGONIAN ANDEAN DEAD \\ ENDS: MEASURING THE INTENSITY OF LAND USE AT THE FRINGES \\ OF THE NORTHERN ICE FIELD (AISÉN, CHILE)
}

Amalia Nuevo-Delaunay², César Méndez ${ }^{2}$, Omar Reyes $^{2,3}$, Andrea Seelenfreund ${ }^{4}$ y Carolina Belmar 5

Las principales vías de circulación en Patagonia, localizadas al este de esta región, generaron que los valles occidentales fueran
marginalmente ocupados, posiblemente visitados de forma estacional y complementaria y, por tanto, habitados con menor inten-
sidad. La idea de la ocupación de los "corredores sin salida de los Andes" de Luis Borrero conforma la plataforma teórica para el
estudio de los valles occidentales de Patagonia. En particular, el área circunscrita por los lagos General Carrera y Cochrane, y por
el Campo de Hielo Norte, corresponde a un excepcional ejemplo de un corredor sin salida. Nuestra investigación arqueológica
se enfoca en la ocupación humana de esta área, a través de la evaluación de la distribución de sitios, cronología y la redundancia
ocupacional, como medio para generar una perspectiva preliminar de un área escasamente estudiada. Los resultados sugieren que
las ocupaciones humanas se extendieron durante los últimos 8600 años, al principio de forma discontinua y después de 3000 cal.
AP, con mayor continuidad. La variabilidad de sitios, la riqueza de los conjuntos y la redundancia a escala de sitio ocurrieron a
la par de la mayor continuidad regional. Estas trayectorias son muy similares a aquellas detectadas al este del área de estudio y
mantienen importantes diferencias con otras cuencas al norte de Patagonia Centro Occidental.

Palabras claves: corredores sin salida, barreras biogeográficas, cazadores recolectores, Holoceno, Patagonia Centro Oeste.

Patagonia's main circulation routes, which were located in the eastern area of this region, fostered a marginal occupation of western valleys, possibly by means of seasonal, complementary visits, resulting in less intense settlements. Luis Borrero's conception of the occupation of "Andean dead ends" is the pivotal framework for the study of Patagonia's western valleys. In particular, the area circumscribed by the General Carrera and Cochrane lakes and the Northern Ice field stands as a remarkable example of a dead end. In this archaeological study, we focus on the human occupation of this area by assessing site distribution, chronology, and occupational redundancy as a way of providing a preliminary outlook on an understudied area. Results suggest that human occupations extended over the last 8600 years, at first discontinuously, and with greater continuity after 3000 cal. BP. Site variability, assemblage richness, and redundancy at a site scale came alongside regional continuity. These trends are remarkably similar to those detected east of the study area and hold important differences from other to the basins north of Central Western Patagonia.

Key words: Andean dead ends, biogeographical barriers, hunter gatherers, Holocene, Central Western Patagonia.

Las principales vías de circulación humana en Patagonia durante el Holoceno se ubicaron en el este, como lo indican la antigüedad del poblamiento inicial, la mayor frecuencia de sitios, las tasas más altas de depositación de material arqueológico, la mayor frecuencia de eventos ocupacionales por cada unidad muestreada y la circulación de las materias primas líticas (Borrero 2004; Borrero et al. 2019;

1 Una primera versión de este trabajo fue presentada en el Simposio Patagonian evolutionary archaeology and human paleoecology: Commending the legacy (still in the making) of Luis Alberto Borrero in the interpretation of hunter-gatherer studies of the Southern Cone, realizado en el marco de la reunión anual de la Society for American Archaeology realizada en Albuquerque, USA (abril 2019). Este manuscrito fue revisado por pares externos y editado por el Comité Editor de Chungara y por uno de los editores invitados, Juan Bautista Belardi.

2 Centro de Investigación en Ecosistemas de la Patagonia, Coyhaique, Chile. amalia.nuevo@ciep.cl; cesar.mendez@ciep.cl

3 Centro de Estudios del Hombre Austral, Instituto de la Patagonia, Universidad de Magallanes, Punta Arenas, Chile. omarreyesbaez@gmail.com

4 Escuela de Antropología, Universidad Academia de Humanismo Cristiano, Santiago, Chile. aseelenfreund@academia.cl

5 Departamento de Antropología, Universidad de Chile, Santiago, Chile. cbelmarp@u.uchile.cl 
Pérez et al. 2016; Prates et al. 2013; Stern 2018). Espacios del occidente de Patagonia, como los valles andinos de Aisén continental, han sido considerados como marginales, ocupados desde los núcleos poblacionales orientales donde la ocupación humana fue más permanente y donde ocurrió la circulación principal de los grupos (Borrero 2004; Méndez, Nuevo Delaunay et al. 2018). Estos valles habrían conformado verdaderos corredores secundarios o vectores para una circulación principalmente este-oeste (Borrero 2004). Estas trayectorias son significativas en tanto el sector occidental de Patagonia desarrolló ecosistemas atractivos para la ocupación humana posiblemente solo hace 12000 años atrás, más de un milenio más tarde que las áreas al este (Borrero et al. 2019; Méndez 2013), y durante el Holoceno fue lugar de ocupaciones menos frecuentes, continuas e intensas que en el oriente (Méndez, De Porras et al. 2016; Nuevo Delaunay et al. 2013). Pese a la menor frecuencia de registro arqueológico esperable en áreas marginales, el estudio de su ocupación es informativo sobre procesos que acontecieron en escalas espaciales más amplias, ya que estas áreas han demostrado ser más sensibles a los cambios en la movilidad y el asentamiento en escalas locales y regionales (Veth 1993). El objetivo principal de este trabajo es evaluar la ocupación humana en uno de los "callejones sin salida" más evidente y menos estudiado en Patagonia: el área al este del Campo de Hielo Norte, circunscrita por los lagos General Carrera-Buenos Aires y Cochrane-Pueyrredón (Figura 1). Se propone caracterizar la distribución del registro arqueológico, la cronología de las ocupaciones y evaluar la redundancia ocupacional a escala de los sitios como medidas para abordar cualitativa y cuantitativamente las trayectorias de ocupación de los márgenes occidentales de Patagonia en el marco de procesos humanos de escala amplia.

\section{Antecedentes}

Geográficamente, distintas áreas andinas de Patagonia pueden considerarse como callejones sin salida, entre ellas: la cuenca del Río Ibáñez, el Parque Nacional Perito Moreno, el área entre el Lago Argentino y la Cordillera Baguales, el área entre los lagos San Martín y Viedma, la "península" entre los lagos Viedma y Argentino, entre otras (Aschero et al. 2005; Belardi

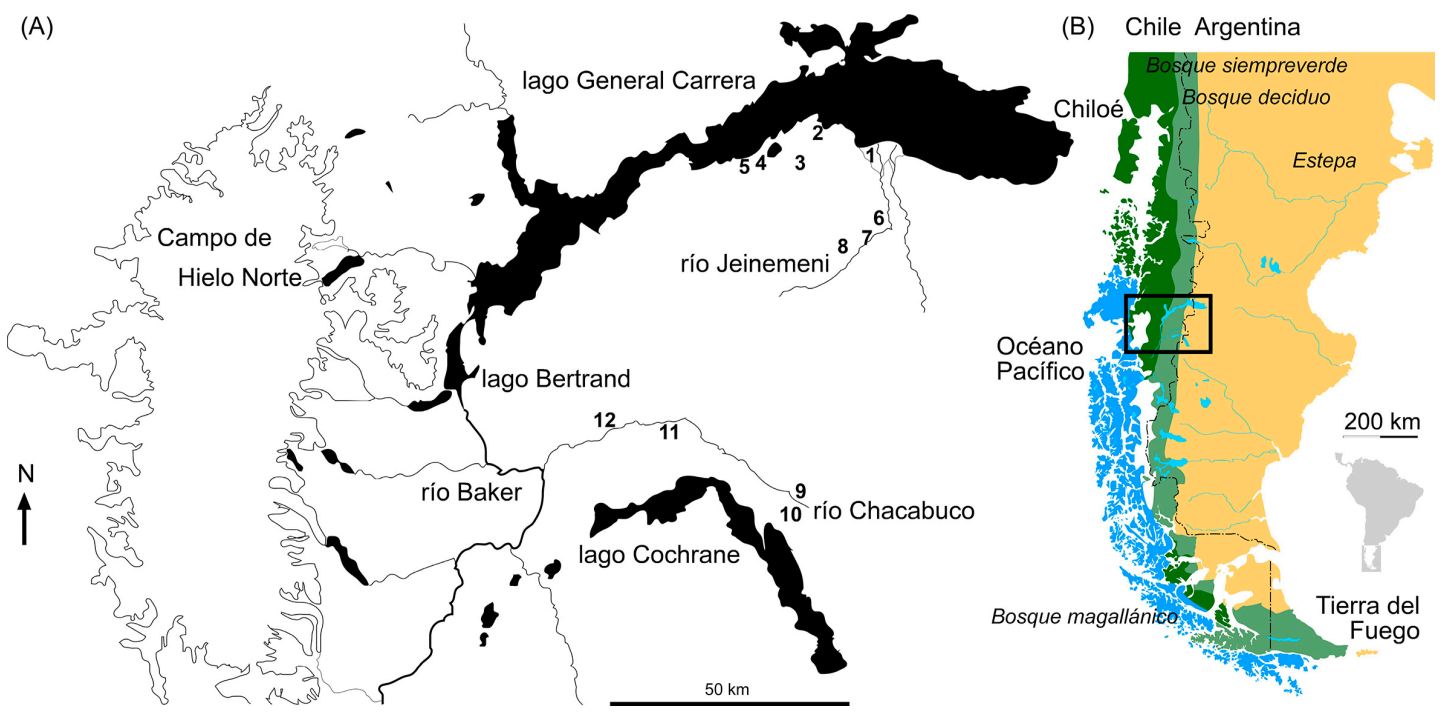

Figura 1. (A) Ubicación del área de estudio y (B) distribución de sitios al este del Campo de Hielo Norte; (1) Chile Chico 1; (2) Chenque Bahía Jara; (3) Pampa La Perra (Chenque y PPS16-17); (4) Laguna El Peligro; (5) La Tina; (6) Bloque Jeinemeni; (7) Quebrada Honda; (8) Cueva de las Manos del Río Pedregoso; (9) Cueva Los Carneros; (10) Alero Entrada Baker y Laguna Alta; (11) Alero Blanco; (12) Alero Gianella.

(A) Location of the study area and (B) distribution of sites east of the Northern Icefield; (1) Chile Chico 1; (2) Chenque Bahía Jara; (3) Pampa La Perra (Chenque and PPS16-17); (4) Laguna El Peligro; (5) La Tina; (6) Bloque Jeinemeni; (7) Quebrada Honda; (8) Cueva de las Manos del Río Pedregoso; (9) Cueva Los Carneros; (10) Alero Entrada Baker and Laguna Alta; (11) Alero Blanco; 
y Carballo Marina 2014; Borrero 2004; Carballo Marina et al. 2016; García y Mena 2016; Pallo y Borrero 2015). Las características de la ocupación de los espacios definidos como callejones sin salida están aún escasamente abordadas arqueológicamente, en comparación con las de los espacios abiertos de estepa y las costas del Atlántico. Para el caso de los valles transversales andinos de Aisén continental, se ha propuesto que los corredores y callejones sin salida fueron poblados desde el oriente, al menos un milenio después que las estepas, de forma marginal y discontinua, complementaria y posiblemente estacional, de modo cuantitativamente decreciente hacia el oeste y sin conectividad con el Pacífico (Belmar et al. 2017; Borrero et al. 2019; Méndez y Reyes 2008; Méndez et al. 2014; Méndez, Reyes et al. 2016; Reyes et al. 2009). Adicionalmente, resultados de investigaciones en distintos valles indican que las ocupaciones en los corredores no fueron iguales, ni invariables a través del tiempo, ya que en cuencas como Cisnes se articuló en la forma de un pulso (3000 a 2000 cal. AP), mientras que en Ibáñez la ocupación fue más sostenida durante los últimos 2000 años (Mena 2000; Méndez y Reyes 2008).

Hasta el momento, los antecedentes de investigación al este del Campo de Hielo Norte se habían centrado en los valles de los ríos Jeinemeni y Chacabuco. Las investigaciones en esta zona, no obstante llevar cerca de 50 años, contaban solo con nueve fechados ${ }^{14} \mathrm{C}$; además de ocho edades experimentales en termoluminiscencia de líticos termoalterados, todos en el Valle de Chacabuco (Mena et al. 2014). Las intervenciones en sitios arqueológicos solo se habían conducido en Alero Entrada Baker, Cueva Los Carneros, Alero Gianella y Alero Blanco, siendo en su mayoría restringidas, inconclusas o preliminarmente reportadas (Fuentes-Mucherl et al. 2012; Mena y Blanco 2017; Mena y Jackson 1991; Mena y López 2014; Méndez y Velásquez 2005). Sobre la base de información de los conjuntos líticos, especialmente los de superficie, se hipotetizó que la ocupación de este valle fue de tipo estacional, con fuertes lazos a los territorios al sureste de acuerdo a la distribución de materias primas (i.e. obsidiana de Pampa del Asador) y probablemente orientada hacia actividades de caza (Méndez 2004; Méndez y Velásquez 2005; Méndez et al. 2004). Respecto a Jeinemeni, la única información disponible corresponde a menciones del arte rupestre (i.e., Cueva de las Manos del Río Pedregoso), los primeros estudios bioantropológicos de la región y una caracterización preliminar de sus conjuntos superficiales en torno a la localidad de Chile Chico (Contreras 2012; Ericksen 1965; Méndez 2004; Niemeyer 1978; Sade et al. 2019).

\section{Área de Estudio}

El área al este del Campo de Hielo Norte comprende el sector limitado por el Lago General Carrera-Buenos Aires por el norte, el Lago CochranePueyrredón por el sur y el Río Baker (el más caudaloso de Chile, con $870 \mathrm{~m}^{3} / \mathrm{s}$ ), junto al mencionado campo glaciar por el oeste $\left(\sim 4.200 \mathrm{~km}^{2}\right)$, con las principales alturas de la región (p.ej., Monte San Valentín, $4.058 \mathrm{msm}$ ). Estas formas del paisaje corresponden a verdaderas barreras geográficas que encauzan el desplazamiento de los seres humanos (Figura 2). La costa sur del Lago General Carrera y el Río Chacabuco se articulan como ejes este-oeste, mientras que el Río Jeinemeni, en el extremo este, escurre de sur a norte, delimitando un amplio sector intermedio correspondiente a cordones subandinos orientales y la meseta del Jeinemeni. El clima del oeste de Patagonia y su variabilidad histórica están principalmente controlados por la posición y fuerza del cinturón de vientos del oeste (CVO) y su interacción con la Cordillera Andina (Garreaud 2009). La presencia del Campo de Hielo en esta latitud genera un marcado efecto orográfico, llegando a una reducción de ocho a 10 veces la pluviosidad en la estepa con relación al área costera (Romero 1985). Consecuentemente, se presenta de oeste a este un gradiente de pisos de vegetación de bosque siempreverde mixto, bosque caducifolio templado, matorral arborescente caducifolio y estepa mediterránea templada (Luebert y Pliscoff 2006).

Durante el Último Máximo Glacial el Campo de Hielo correspondía a una gran masa glaciar, fusionada sobre la cordillera y áreas vecinas, y cuyos principales drenajes ocurrían hacia el Atlántico (Davies et al. 2020). Investigaciones regionales muestran un temprano inicio de la deglaciación (19000-18000 años cal AP.), generándose grandes sistemas lacustres que, hace ca. 14200 años, se acoplaron en cotas bajas, tanto en el norte como en el centro-sur de la región (García et al. 2019; Thorndycraft et al. 2019). Los registros de polen de lagos y pantanos andinos muestran que este proceso estuvo dominado por condiciones frías hasta hace ca. 14800 años atrás, cuando se incrementaron la humedad efectiva y las precipitaciones (De Porras et al. 2012; Henríquez et al. 2017). El desarrollo de los bosques se inició hace 11500 años atrás, producto del incremento en la humedad efectiva y las temperaturas 


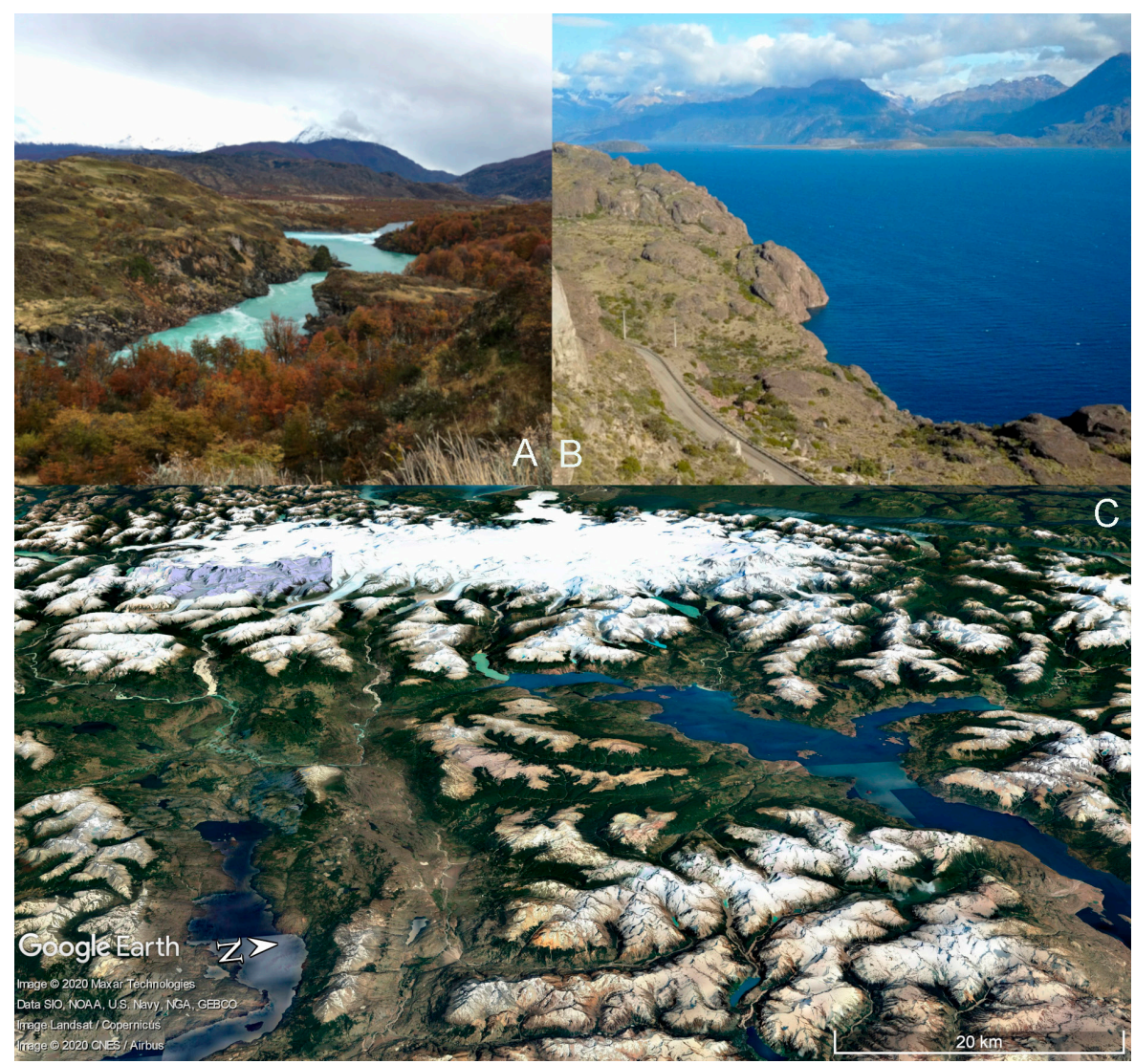

Figura 2. Barreras geográficas del área de estudio. (A) Río Baker, (B) Lago General Carrera, (C) Campo de Hielo Norte, Lago Cochrane y Lago General Carrera (imagen de Google Earth 2020).

Geographic barriers of the study area. (A) Baker river, (B) General Carrera lake, (C) Northern Icefield, Cochrane lake, and General Carrera lake (Google Earth image, 2020).

estivales a causa del debilitamiento del CVO (De Porras et al. 2014; Villa-Martínez et al. 2012;). Hace 8000 años se desarrollaron las máximas extensiones orientales del bosque, junto con un incremento en la densidad del dosel, lo que ocurrió en sincronía con la retirada de hielo en sectores a ca. $1.000 \mathrm{msm}$ (De Porras et al. 2012, 2014; McCulloch et al. 2017). Los registros más orientales y distales del bosque (i.e., más sensibles) muestran hacia 3500-3000 años cal. AP una mayor variabilidad regional (De Porras et al. 2012; McCulloch et al. 2017). Sincrónicamente, se observan mayores acumulaciones de carbón y episodios de fuego de mayor magnitud, los que han sido atribuidos a efectos humanos sobre los patrones de fuego (McCulloch et al. 2017; Méndez, De Porras et al. 2016).

\section{Material y Método}

Este trabajo, por una parte, se basó en un muestreo exhaustivo de material fechable de colecciones procedentes de excavaciones realizadas en investigaciones anteriores y de las cuales no se contaba con datos cronométricos o bien Estos eran insuficientes. Por otra parte, nuevos datos fueron incorporados a partir de investigaciones en el área de estudio que buscan abordar sistemáticamente la intensidad de uso del espacio a partir de distinguir las ocupaciones humanas y su redundancia. El relevamiento inicial de datos arqueológicos en terreno incluyó información procedente de prospecciones superficiales sistemáticas (Contreras 2012; Mena y Blanco 2017; Mena y Lucero 2004). Estas fueron 
revisadas en terreno para verificar la localización de algunos de los puntos de interés arqueológico que no estaban georreferenciados.

Se llevaron a cabo excavaciones en sitios seleccionados. Las excavaciones buscaron reconocer unidades estratigráficas distintivas a través de niveles artificiales de cinco o $10 \mathrm{~cm}$ dependiendo de su resolución estratigráfica y de los contextos, fueran estos a cielo abierto o reparos rocosos. Se procuró comprender las relaciones horizontales, identificar rasgos y registrar todos los artefactos $>2 \mathrm{~cm}$ con ubicación tridimensional. Se incluyó un muestreo sistemático de sedimentos para caracterización geoarqueológica y para flotación. Todos los sedimentos fueron tamizados con malla de $2 \mathrm{~mm}$. Se procuró obtener edades absolutas con técnica AMS para los sitios y contextos discutidos, buscando entender con ellas la sucesión estratigráfica (Tabla 1). Los resultados de edades ${ }^{14} \mathrm{C}$ se calibraron a $2 \sigma$ con el programa Calib 7.0.4 usando la curva ShCal 13 (Hogg et al. 2013; Stuiver et al. 2018). Con el fin de evaluar la redundancia ocupacional, se procedió a combinar las edades estadísticamente indiferenciables $(\alpha=0,05)$ en la escala del sitio arqueológico, las que fueron consideradas como eventos ocupacionales mínimos (Méndez 2013; Ward y Wilson 1978). Los materiales arqueológicos de superficie y de excavación se describen de forma general para realizar una caracterización inicial de los conjuntos presentes. Adicionalmente, se llevó a cabo un muestreo por raspado directo en húmedo de las superficies de implementos de molienda y de soportes líticos para determinar la presencia de microfósiles contenidos en los residuos de uso (Belmar 2019). Se trabajó desde un enfoque de análisis múltiple, comparando con morfotipos de ICPN e ICSN (Coil et al. 2003; ICSN 2011; Madella et al. 2005).

\section{Resultados}

\section{Costa sur del Lago General Carrera}

Chile Chico 1 (RJ-80; 4632'4.8" S; 71²41'72.9"' W; $210 \mathrm{msm}$ ) corresponde a un sitio a cielo abierto al interior de la localidad de Chile Chico, ubicado al oeste de la desembocadura del Río Jeinemeni, sobre una duna de arenas a orillas del Lago General Carrera. El sitio muestra materiales arqueológicos dispersos en la superficie, entre los que se observó abundante cantidad de desechos de talla lítica, algunos instrumentos formales, escasos fragmentos de cerámica y ocasionales implementos de molienda. Los restos cerámicos se encuentran altamente fragmentados, tienen las paredes alisadas interior y exterior de tonos de grises con cocción oxidante incompleta. Algunos fragmentos presentan ahumado en las superficies interiores, lo que sugiere posibles procesos de carbonización en la parte interna de las piezas. Los análisis de residuos de una mano de moler registrada en superficie arrojaron la presencia de granos de almidón de Mutisia spinosa (clavel del campo) y de Solanum Tuberosum (papa), ambos con daños producto de la molienda. Dos concentraciones superficiales de carbón de gran tamaño $\left(>1 \mathrm{~m}^{2}\right)$ que fueron expuestas por trabajos de remoción de sedimentos anteriores a nuestra investigación se encontraban distantes unos $8 \mathrm{~m}$ entre sí. Dos fechados ${ }^{14} \mathrm{C}$ fueron obtenidos de cada una de ellas con edades resultantes en 290440 cal. AP y 30-500 cal. AP (Tabla 1). Estas son estadísticamente diferentes, por lo que no representan un mismo evento de quema.

A fin de caracterizar la expresión estratigráfica del depósito, se llevó a cabo una red de barrenos, la que permitió seleccionar un área para una excavación de $4 \mathrm{~m}^{2}$ a una distancia aproximada de 20-30 m de los fogones expuestos, donde se obtuvieron los fechados. La excavación mostró una unidad estratigráfica (UE) superior de arena friable, que suprayace en forma difusa a una unidad de arena más fina y compacta. El material arqueológico se concentra en los primeros $10 \mathrm{~cm}$. El conjunto lítico está compuesto por 93 piezas, la mayoría $(\mathrm{N}=89)$ correspondientes a lascas, completas en un $42 \%$. Solo se registró un instrumento tallado y un guijarro con pigmento. Predominan fuertemente las rocas silíceas $(87,1 \%)$, registrándose además especímenes de toba cinerítica, andesita y una pequeña lasca de retoque de obsidiana. El conjunto óseo de Chile Chico 1 se registra altamente carbonizado y fragmentado, lo que no permite su caracterización (NISP=42).

El sitio Pampa La Perra (PPS16-17; 46 33'33.6" S; 71 54 '54.3" W; 499 msm), ubicado en el margen sur de la pampa homónima, corresponde a un área circunscrita entre un alero de $30 \mathrm{~m}$ de largo, que ofrece protección de los vientos del noreste y del sur, y un bloque desprendido distante a ca. $15 \mathrm{~m}$ en dirección norte (Figura 3a). La superficie se encuentra cubierta por una capa de ceniza de unos $20 \mathrm{~cm}$ de espesor y presenta escaso material lítico en superficie, consistente principalmente en desechos de talla de rocas silíceas. Tres sondeos de $0,09 \mathrm{~m}^{2} \mathrm{se}$ usaron para caracterizar la distribución subsuperficial 
Tabla 1. Edades radiocarbónicas de sitios arqueológicos al este del Campo de Hielo Norte. Radiocarbon dates of archaeological sites east of the Northern Icefield.

\begin{tabular}{|c|c|c|c|c|c|}
\hline Sitio & Referencias espaciales & Código laboratorio & edad $14 \mathrm{C}$ & calibración $2 \sigma$ & Material \\
\hline \multirow{2}{*}{ Chile Chico 1} & Fogón \#1; superficie & D-AMS 027551 & $309 \pm 20 \mathrm{AP}$ & 290-440 cal. AP & carbón \\
\hline & Fogón \#2; superficie & D-AMS 027552 & $413 \pm 27 \mathrm{AP}$ & $330-500 \mathrm{cal}$. AP & carbón \\
\hline Chenque Bahía Jara & Chenque & D-AMS 027557 & $571 \pm 24$ AP & $510-560 \mathrm{cal}$. AP & colágeno óseo \\
\hline $\begin{array}{l}\text { Chenque Pampa La } \\
\text { Perra }\end{array}$ & Individuo 1 (colección) & D-AMS 029044 & $707 \pm 25 \mathrm{AP}$ & 560-670 cal. AP & colágeno dental \\
\hline \multirow{2}{*}{ Pampa La Perra } & U.1; UE1; 30-35 cm & D-AMS 027549 & moderno & moderno & carbón \\
\hline & U.1; UE1; 70-75 cm & D-AMS 027550 & $1204 \pm 28$ AP & $970-1180 \mathrm{cal} . \mathrm{AP}$ & carbón \\
\hline Laguna El Peligro & Perfil expuesto & D-AMS 032606 & $3361 \pm 31 \mathrm{AP}$ & $3460-3640 \mathrm{cal}$. AP & colágeno óseo \\
\hline Bloque Jeinemeni & U.1D-E; UE1; 20-30 cm & D-AMS 027558 & moderno & moderno & colágeno óseo \\
\hline \multirow{5}{*}{ Quebrada Honda } & Perfil A; U.1; UE2; 5-10 cm & D-AMS 032596 & $481 \pm 24$ AP & $470-530 \mathrm{cal}$. AP & carbón \\
\hline & Perfil A; U.1; UE3; 55-60 cm & D-AMS 032597 & $1968 \pm 35 \mathrm{AP}$ & 1810-2000 cal. AP & carbón \\
\hline & Perfil A; UE3 (medio) & UGAMS 39294 & $2200 \pm 20 \mathrm{AP}$ & 2090-2310 cal. AP & colágeno óseo \\
\hline & Perfil B; UE1(techo) & D-AMS 032605 & $1208 \pm 29$ AP & 980-1180 cal. AP & colágeno óseo \\
\hline & Perfil B; UE1 (medio) & D-AMS 027553 & $1697 \pm 23 \mathrm{AP}$ & 1510-1610 cal. AP & carbón \\
\hline \multirow{17}{*}{ Cueva Los Carneros } & U.7G; UE3; 41-50 cm & D-AMS 030084 & $230 \pm 26 \mathrm{AP}$ & $150-300 \mathrm{cal}$. AP & carbón \\
\hline & U.7E; UE3; 31-40 cm & D-AMS 030085 & $422 \pm 25 \mathrm{AP}$ & $330-500 \mathrm{cal}$. AP & carbón \\
\hline & U.7E; UE3; 41-50 cm & D-AMS 030086 & $876 \pm 26 \mathrm{AP}$ & 680-780 cal. AP & carbón \\
\hline & U.7G; UE3; 71-80 cm & D-AMS 030083 & $1009 \pm 26 \mathrm{AP}$ & $800-930$ cal. AP & carbón \\
\hline & U.6F; UE3; $126 \mathrm{~cm}$ & D-AMS 034574 & $1284 \pm 27 \mathrm{AP}$ & 1070-1270 cal. AP & carbón \\
\hline & U.7F; UE3b; $145 \mathrm{~cm}$ & UGAMS $8791^{\mathrm{a}}$ & $1285 \pm 25 \mathrm{AP}$ & 1070-1270 cal. AP & carbón \\
\hline & U.7F; UE4; $135 \mathrm{~cm}$ & D-AMS 034575 & $1285 \pm 27 \mathrm{AP}$ & $1070-1270 \mathrm{cal} . \mathrm{AP}$ & carbón \\
\hline & U.5C; UE1; 50-60cm & UGAMS $11756^{\mathrm{b}}$ & $1490 \pm 50 \mathrm{AP}$ & $1270-1430 \mathrm{cal} . \mathrm{AP}$ & colágeno óseo \\
\hline & U.7F; UE4; $144 \mathrm{~cm}$ & D-AMS 034576 & $1548 \pm 25 \mathrm{AP}$ & 1310-1430 cal. AP & carbón \\
\hline & U.7G; UE4; 140-150 cm & D-AMS 032601 & $1595 \pm 25 \mathrm{AP}$ & $1370-1520 \mathrm{cal}$. AP & carbón \\
\hline & U.7F; UE4; $159 \mathrm{~cm}$ & D-AMS 034578 & $1857 \pm 28 \mathrm{AP}$ & 1700-1830 cal. AP & carbón \\
\hline & U.7F; UE4; $154 \mathrm{~cm}$ & D-AMS 034577 & $1972 \pm 27$ AP & 1820-1930 cal. AP & carbón \\
\hline & U.6G; UE4-5; 150-155 cm & D-AMS 032602 & $2142 \pm 29 \mathrm{AP}$ & 2000-2150 cal. AP & carbón \\
\hline & U.6G; UE5; $168 \mathrm{~cm}$ & D-AMS 034580 & $2430 \pm 28 \mathrm{AP}$ & 2340-2680 cal. AP & carbón \\
\hline & U.6G; UE5; $175 \mathrm{~cm}$ & D-AMS 034581 & $2490 \pm 28 \mathrm{AP}$ & 2360-2700 cal. AP & carbón \\
\hline & U.7F; UE6-7; $184 \mathrm{~cm}$ & D-AMS 034583 & $2894 \pm 30 \mathrm{AP}$ & 2860-3070 cal. AP & carbón \\
\hline & U.7G; UE6; $179 \mathrm{~cm}$ & D-AMS 034582 & $2917 \pm 30 \mathrm{AP}$ & 2880-3110 cal. AP & carbón \\
\hline \multirow{10}{*}{$\begin{array}{l}\text { Alero Entrada } \\
\text { Baker }\end{array}$} & Talud; Barreno \#1; 115-125 cm & D-AMS 027555 & moderno & moderno & carbón \\
\hline & Interior; S/D; UE1; 30-40 cm & BETA $21928^{c}$ & $230 \pm 70 \mathrm{AP}$ & $1-390 \mathrm{cal}$. AP & carbón \\
\hline & Interior; U.11C; S/D; 50-60 cm & D-AMS 027554 & $323 \pm 27$ AP & $300-450 \mathrm{cal}$. AP & carbón \\
\hline & Talud; U.2B; UE1; $20-30 \mathrm{~cm}$ & D-AMS 011589 & $343 \pm 23 \mathrm{AP}$ & $300-450 \mathrm{cal}$. AP & carbón \\
\hline & Interior; S/D; UE1; 40-50 cm & BETA $113797^{d}$ & $410 \pm 50 \mathrm{AP}$ & $320-500 \mathrm{cal}$. AP & carbón \\
\hline & Interior; U.8C; S/D; 70-80 cm & D-AMS 027560 & $1273 \pm 24 \mathrm{AP}$ & $1070-1240 \mathrm{cal} . \mathrm{AP}$ & colágeno óseo \\
\hline & Talud; 1-2 A-B; UE1; 70-80 cm & BETA $113796^{\mathrm{d}}$ & $2100 \pm 40 \mathrm{AP}$ & 1930-2120 cal. AP & carbón \\
\hline & Interior; U.12B; UE1; 30-40 cm & UCLA $2712 b^{c}$ & $2580 \pm 50 \mathrm{AP}$ & $2430-2760 \mathrm{cal}$. AP & carbón \\
\hline & Talud; U.2A-B; S/D; $150-160 \mathrm{~cm}$ & D-AMS 027561 & $4451 \pm 29$ AP & $4860-5220 \mathrm{cal}$. AP & colágeno óseo \\
\hline & Interior; U.11C; UE1; 60-70 cm & UCLA $2712 \mathrm{a}^{\mathrm{c}}$ & $7750 \pm 110 \mathrm{AP}$ & $8310-8780 \mathrm{cal} . \mathrm{AP}$ & carbón \\
\hline \multirow{2}{*}{ Laguna Alta } & U.1; UE1B; $40 \mathrm{~cm}$ & D-AMS 032598 & $789 \pm 28$ AP & $660-730 \mathrm{cal} . \mathrm{AP}$ & carbón \\
\hline & Perfil expuesto; UE1; $40 \mathrm{~cm}$ & D-AMS 027562 & $2238 \pm 27 \mathrm{AP}$ & $2150-2320 \mathrm{cal} . \mathrm{AP}$ & colágeno óseo \\
\hline \multirow{4}{*}{ Alero Gianella } & U.1A; UE3 & D-AMS 030087 & $470 \pm 27 \mathrm{AP}$ & $450-520 \mathrm{cal} . \mathrm{AP}$ & carbón \\
\hline & U.2B; UE4 & D-AMS 030089 & $2352 \pm 27 \mathrm{AP}$ & 2180-2380 cal. AP & carbón \\
\hline & U.1A; UE5 & UGAMS 5867 & $2710 \pm 50 \mathrm{AP}$ & 2720-2880 cal. AP & carbón \\
\hline & U.1A; UE5 & D-AMS 030088 & $3698 \pm 41 \mathrm{AP}$ & $3850-4090 \mathrm{cal}$. AP & carbón \\
\hline \multirow{3}{*}{ Alero Blanco } & U.1; UE1; 6-10 cm & D-AMS 030090 & $448 \pm 25 \mathrm{AP}$ & $340-510 \mathrm{cal}$. AP & carbón \\
\hline & U.2; UE1; 5 cm & UGAMS $6220^{\mathrm{f}}$ & $1170 \pm 25 \mathrm{AP}$ & $960-1060 \mathrm{cal}$. AP & carbón \\
\hline & U.1; UE1; 16-20 cm & D-AMS 030091 & $1268 \pm 27 \mathrm{AP}$ & $1070-1260 \mathrm{cal} . \mathrm{AP}$ & carbón \\
\hline
\end{tabular}

Nota: Todas las edades son inéditas; excepto a. Mena y López 2014, b. Prieto y Mena 2016, c. Mena y Jackson 1991, d. Méndez y Velásquez 2005, e. Fuentes et al. 2012, f. Mena y Blanco 2017.

del registro arqueológico que profundiza hasta un máximo de $90 \mathrm{~cm}$. La estratigrafía de techo a base muestra una unidad de limos arenosos que expone una mayor proporción de clastos en la medida que se profundiza. El sondeo emplazado al este (U.2) mostró una mayor proporción de clastos a lo largo del depósito, sugiriendo que corresponde a un área de mayor energía. Un fechado ${ }^{14} \mathrm{C}$ de la base de la excavación de la unidad 1 arrojó una edad de 9701180 cal. AP, mientras que un fechado de la parte superior de la misma resultó en una edad moderna.

En vista de lo restringido de las excavaciones, se consideró en conjunto el registro de las tres unidades excavadas. Los materiales líticos ( $\mathrm{N}=199)$ se distribuyen 

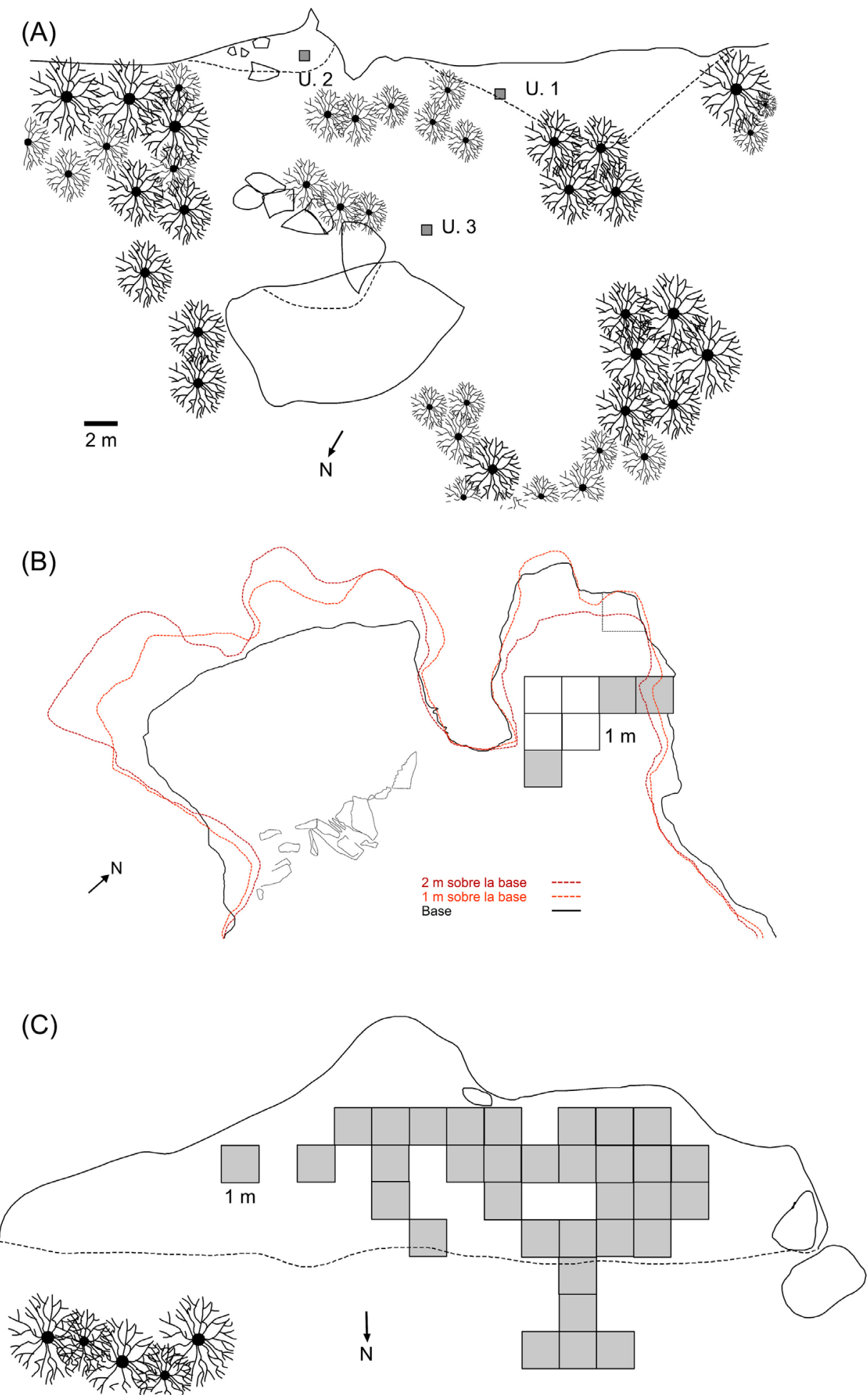

Figura 3. Vista de plantas de sitios seleccionados: (A) Pampa la Perra, (B) Cueva Los Carneros, (C) Alero Entrada Baker (basado en Mena y Jackson 1991). Cuadrículas en gris: excavadas en investigaciones anteriores, en blanco excavadas en esta investigación. Plan view of selected sites: (A) Pampa La Perra, (B) Cueva Los Carneros, (C)Alero Entrada Baker (based on Mena and Jackson 1991). Quadrats in grey: Excavated in previous studies. Quadrats in white: excavated in this study. 
con relativa homogeneidad a lo largo del depósito y corresponden en un 56\% a rocas silíceas (18\% son posiblemente de origen local, sobre la base de observaciones preliminares a la distribución de recursos líticos) y $18 \%$ a obsidiana. La secuencia de reducción está dominada por desechos de talla de diversas etapas $(79 \%)$ y no se registraron instrumentos propiamente tales. Respecto a la fauna (NISP=54), solo se pudieron identificar nueve especímenes, entre ellos fragmentos de Ovis aries, Lama guanicoe, Rhea pennata y especies de roedores. En general se encuentran con baja fragmentación y evidencias de combustión en un $23 \%$.

En el sector noreste de la Pampa de la Perra, a $1 \mathrm{~km}$ del sitio anterior, existe referencia del hallazgo de varios chenques (BJ-4), donde fueron obtenidos los restos de al menos un individuo (Mena 1987). Fruto de ésta y otras intervenciones no documentadas, existen restos bioantropológicos depositados en la Casa de la Cultura de la Municipalidad de Chile Chico. Como parte del muestreo realizado, se seleccionó un cráneo para el que se obtuvo una fecha directa de un incisivo que arrojó una edad de 560-670 cal. AP. Resultados de isótopos estables sobre un tercer molar del mismo individuo arrojaron valores de $\delta^{13} \mathrm{C}=-19,16 \% ; \delta^{15} \mathrm{~N}=$ $11,13 \% ; \mathrm{C} / \mathrm{N}=3,24$ (UGAMS 40946). Esta datación concuerda con un segundo chenque (BJ-2), proveniente de la localidad de Bahía Jara (Reyes 2004), el que arrojó una edad de 510-560 cal. AP.

Laguna El Peligro (RJ-81; 46³3'47.2"' S; $72^{\circ} 00^{\prime} 37.8^{\prime \prime} \mathrm{W} ; 472 \mathrm{msm}$ ) corresponde a un sitio a cielo abierto en la margen este de una laguna estacional, a 2,5 km al oeste de Laguna Verde. Evidencias óseas y líticas se distribuyen en una superficie de $1.800 \mathrm{~m}^{2}$. Observaciones al área de mayor concentración sugieren que las evidencias provienen de una UE arenosa desde donde se exponen por la acción conjunta entre raíces de Schinus y el tránsito del ganado vacuno. Preliminarmente, los materiales óseos tienen una meteorización semejable al estadio 2 de Behrensmeyer (1978). Los materiales líticos se registran en menor proporción e incluyen grandes lascas de andesita y desechos de obsidiana negra en baja proporción. Análisis de residuos de dos soportes líticos registrados en superficie no arrojaron microfósiles. Un fechado de un fragmento de hueso largo de $L$. guanicoe con huellas de un lascado intencional arrojó una edad de 3460-3640 cal. AP. Esta constituye una fecha preliminar para el conjunto superficial en espera de una evaluación estratigráfica.
Una de las manifestaciones humanas más característica de la costa sur del Lago General Carrera corresponde a su arte rupestre. Los sitios conocidos se concentran principalmente en el área entre Paso Las Llaves y Laguna Verde. Entre ellos destaca el sitio La Tina (RJ-82; 46³3'25.6" S; 7203'03.1", $\mathrm{W} ; 400 \mathrm{msm}$ ), donde se ha registrado la más alta concentración de negativos de mano $(\mathrm{N} \geq 65)$ con el color rojo como tonalidad dominante (Figura 4a). Corresponde a un área reparada (ca. $60 \mathrm{~m}^{2}$ ) por un alto paredón de exposición noreste, en donde se destacan seis paneles con pinturas rupestres. Una unidad de excavación de $2 \mathrm{~m}^{2}$ se utilizó para caracterizar la estratigrafía del sitio y evaluar la presencia de material arqueológico y rasgos asociados en el depósito. De techo a base se observa una unidad superior de matriz arenosa gruesa a grava correspondiente a cenizas de la erupción del Volcán Hudson (1991) que suprayace a una UE de limo con presencia variable de clastos producto de la meteorización in situ de la roca de caja. Los materiales arqueológicos están contenidos principalmente en la parte inferior de la segunda UE y su presencia se extiende hasta el contacto con la roca madre. Estos incluyen líticos (desechos de talla), fragmentos óseos pequeños y carbón en baja proporción, los que se suman a rasgos de fogones. A la fecha no se cuenta con edades ${ }^{14} \mathrm{C}$ para este sitio.

\section{Cuenca oeste del Río Jeinemeni}

A lo largo del sector oeste del Río Jeinemeni, desde Chile Chico y hasta $45 \mathrm{~km}$ al sur, se han registrado una serie de sitios que se suman a nuevos hallazgos realizados en el marco de nuestras prospecciones (Contreras 2012; Niemeyer 1978). Entre ellos destaca un conspicuo bloque errático denominado Bloque Jeinemeni ( $46^{\circ} 41^{\prime} 29.7^{\prime \prime} \mathrm{S}$; $71^{\circ} 41^{\prime} 45.4^{\prime \prime} \mathrm{W}$; $736 \mathrm{msm}$ ), donde se llevó a cabo una excavación de $1 \mathrm{~m}^{2}$. Arrojó una proporción muy baja de material lítico y escasos restos óseos, uno de los cuales fue fechado y arrojó una edad moderna.

Quebrada Honda (RJ-74; 46²2'25.6"' S; 71 ${ }^{\circ} 42^{\prime} 14.9^{\prime \prime} \mathrm{W} ; 499 \mathrm{msm}$ ) corresponde a un sitio a cielo abierto en la intersección de la quebrada homónima y la ruta X-753, la que corre paralela al Río Jeinemeni. Su construcción expuso dos perfiles que muestran material lítico, óseo y carbón en estratigrafía. Se exponen también desechos de talla lítica y restos óseos meteorizados dispersos en la superficie al sur de la ruta. A fin de caracterizar la expresión estratigráfica 


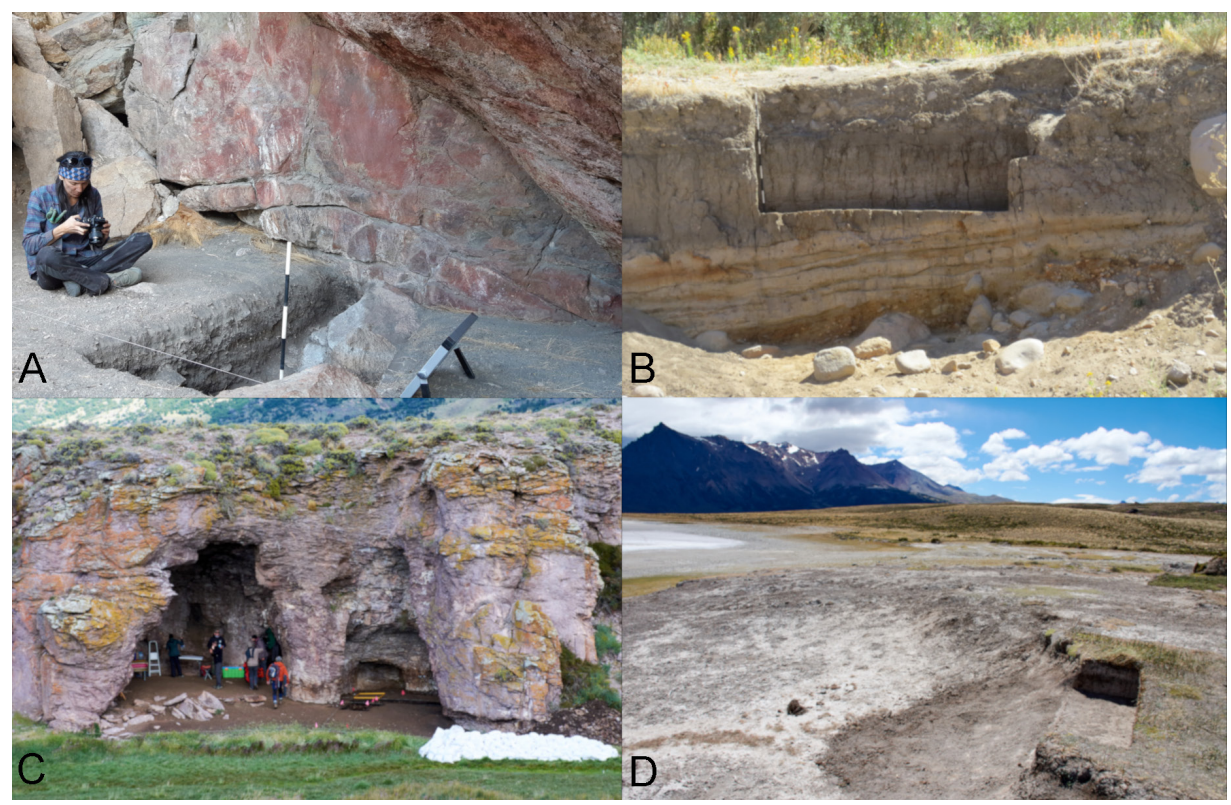

Figura 4. Imágenes de sitios seleccionados del área al este del Campo de Hielo Norte. (A) La Tina, (B) Quebrada Honda (perfil A), (C) Cueva Los Carneros, (D) Laguna Alta.

Images of selected sites from the area east of the Northern Icefield. (A) La Tina, (B) Quebrada Honda (section A), (C) Cueva Los Carneros, (D) Laguna Alta.

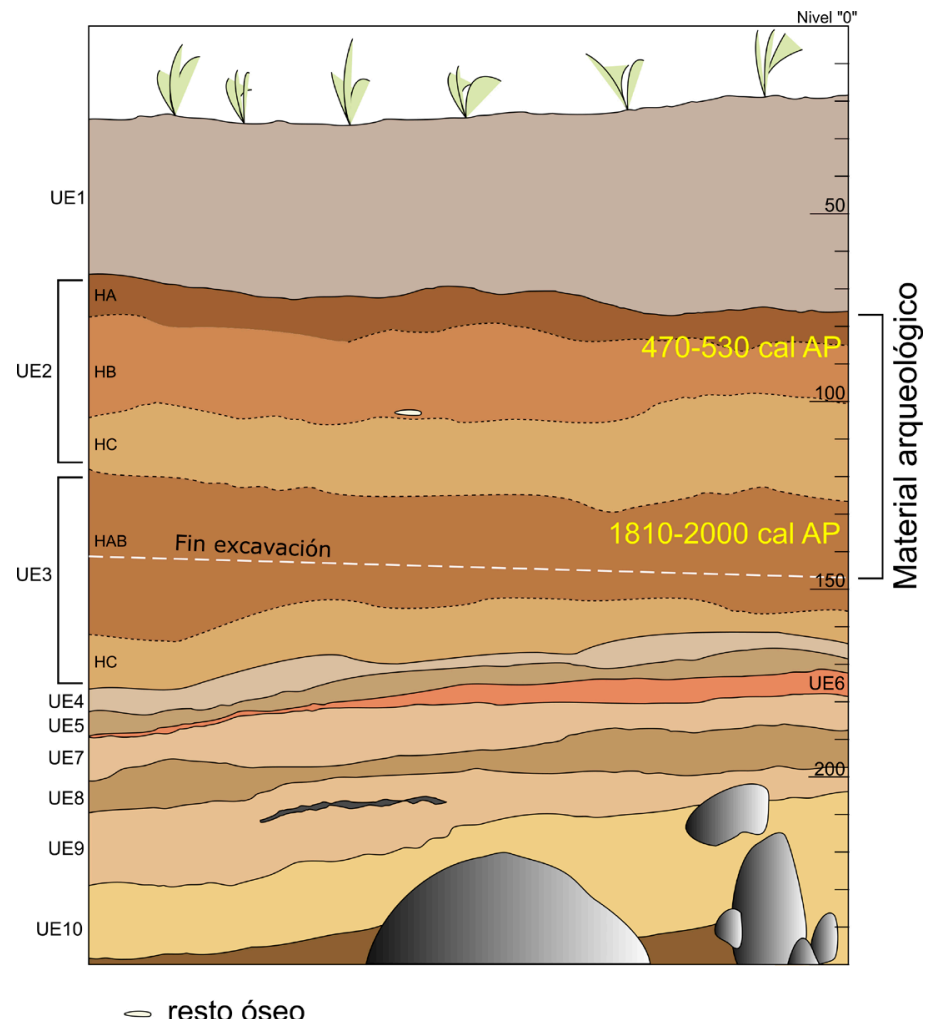

Figura 5. Perfil estratigráfico A del sitio Quebrada Honda. UE: unidad estratigráfica, H: horizonte de suelo, escala vertical en $\mathrm{cm}$.

Stratigraphic section A of the Quebrada Honda site. UE: stratigraphic unit, H: soil horizon, vertical scale in cm. 
del depósito arqueológico, se llevó a cabo una excavación de $1 \mathrm{~m}^{2}$ en un perfil de exposición sur denominado A (Figura 4B). La estratigrafía muestra una UE superior de limo arcilloso con gravilla de origen coluvial que suprayace a una secuencia aluvial donde alternan unidades de limos y arenas ( $\mathrm{N}=8 \mathrm{UE})$ con baja proporción de clastos (5-1\%) (Figura 5). La excepción la constituye la unidad más profunda visible (UE10), compuesta por gravas y abundantes clastos (ocasionalmente clastos sostenidos). Las UE son en general compactas y todos los contactos son netos. Solo las UE 2 y 3 mostraron desarrollo de horizontes de suelo y un contacto difuso entre ambas. El material arqueológico se distribuye desde el techo de UE2 y hasta los $15 \mathrm{~cm}$ superiores de UE3, a lo largo de ca. 65 $\mathrm{cm}$. Una fecha radiocarbónica a ca. $10 \mathrm{~cm}$ del techo arrojó una edad de 470-530 cal. AP, mientras que una muestra ca. $5 \mathrm{~cm}$ sobre el fin del depósito arrojó una edad de 1810-2000 cal. AP. Esta última está en relativo acuerdo con una muestra ósea de otro sector del mismo perfil en similar posición estratigráfica, que arrojó una edad de 2090-2310 cal. AP. Este conjunto de edades también concuerda (e intercaladas) con fechados obtenidos en la secuencia análoga denominada perfil este), donde los resultados de muestras expuestas del techo y medio del depósito son de 980-1180 cal. AP y $1510-1610 \mathrm{cal}$. AP, respectivamente. En total tres edades son de carbón y dos de colágeno de hueso. Otros intentos de fechado sobre hueso resultaron en colágeno insuficiente o bien en resultados con altos valores de $\mathrm{C} / \mathrm{N}$, lo que sugiere degradación del colágeno óseo en el contexto ${ }^{1}$. Todas las edades de este contexto son estadísticamente diferentes entre sí, por lo que indican diferentes eventos ocupacionales a lo largo de 1700 años de formación del depósito estratigráfico.

En la excavación de la unidad 1 el material arqueológico se distribuyó de forma relativamente homogénea a lo largo de la secuencia. Si bien el material se encuentra aún en estudio, es posible señalar que los líticos son los más abundantes. Estos corresponden a lascas de rocas silíceas de calidades medias a buenas, rocas volcánicas basálticas y escasas evidencias de obsidiana. En cuanto a los materiales óseos, se observaron fragmentos de huesos largos y dientes, destacándose la presencia de Lama guanicoe entre las evidencias diagnósticas. Los restos de carbón se encuentran escasamente representados.

Un conjunto de sitios con manifestaciones de arte rupestre había sido descrito en asociación a un sendero del sector Jeinemeni del Parque Nacional
Patagonia administrado por CONAF. Entre ellos destaca el yacimiento denominado Cueva de las Manos del Río Pedregoso (RJ-95; 4643'24" S; 7146'25.6" $\mathrm{W} ; 1.124 \mathrm{msm}$ ), que fuera publicado por Niemeyer (1978). Incluye un área protegida de ca. $40 \mathrm{~m}^{2}$ y en sus paredes se exhiben numerosas superposiciones que totalizan 152 motivos claros (más 61 motivos poco definidos). Representan variados estilos, entre los que se cuentan escenas de caza de camélidos de dimensiones pequeñas, representaciones individuales de camélidos de gran tamaño, negativos de manos, motivos abstractos, zoomorfos y antropomorfos. Una pequeña excavación de $0,5 \mathrm{~m}^{2}$ se usó para caracterizar preliminarmente la secuencia estratigráfica. De techo a base se observa una unidad superior de arenas gruesas $(2-3 \mathrm{~cm})$, friable e inorgánica, correspondiente a cenizas de la erupción del Volcán Hudson (1991). Suprayace a un limo arcilloso gris oscuro (UE2; $15 \mathrm{~cm}$ ), semifriable y orgánico, con presencia de guano de oveja y donde se desarrollan rasgos de quema superpuestos (posiblemente extendidos). Discontinuamente, sobre la roca madre se desarrollan limos color marrón oscuro, inorgánicos $(2-3 \mathrm{~cm})$. Los materiales arqueológicos se registraron entre el fin de la UE2 y hasta el contacto con la roca madre. Estos incluyen únicamente microlascas líticas (desechos de talla), fragmentos óseos pequeños y carbón en baja proporción. A la fecha no se cuenta con edades ${ }^{14} \mathrm{C}$ para este sitio y el estudio del arte rupestre está en proceso.

\section{Cuenca del Río Chacabuco}

La tercera área que concentra sitios fechados corresponde a la cuenca del Río Chacabuco. En el sector de estepa oriental nuestras intervenciones se concentraron en el sitio Cueva Los Carneros ( $\mathrm{RCH03}$; 4709'49.7” S; 71 ${ }^{\circ} 54^{\prime} 15.2^{\prime \prime}$ W; 624 msm, Figura 4C). La cueva se compone de dos cámaras y ofrece un reparo de $57 \mathrm{~m}^{2}$ (Figura 3B). Excavado anteriormente por F. Mena en los años 2000 y 2011, sus intervenciones no profundizaron más allá de las UE superiores (Mena y López 2014). Nuevas excavaciones $\left(5 \mathrm{~m}^{2}\right)$ en la cámara al este han permitido precisar la cronología del sitio y han revelado una secuencia estratigráfica horizontalmente ordenada que cubre con precisión los últimos 3000 años (Figura 6). De techo a base se observa una UE superior de estiércol de oveja que se dispone de forma continua, sellando el depósito con ca. 20-40 $\mathrm{cm}$ de espesor, a la que le sigue discontinuamente una UE arcillosa de escasos $5 \mathrm{~cm}$. Por debajo se expresa 


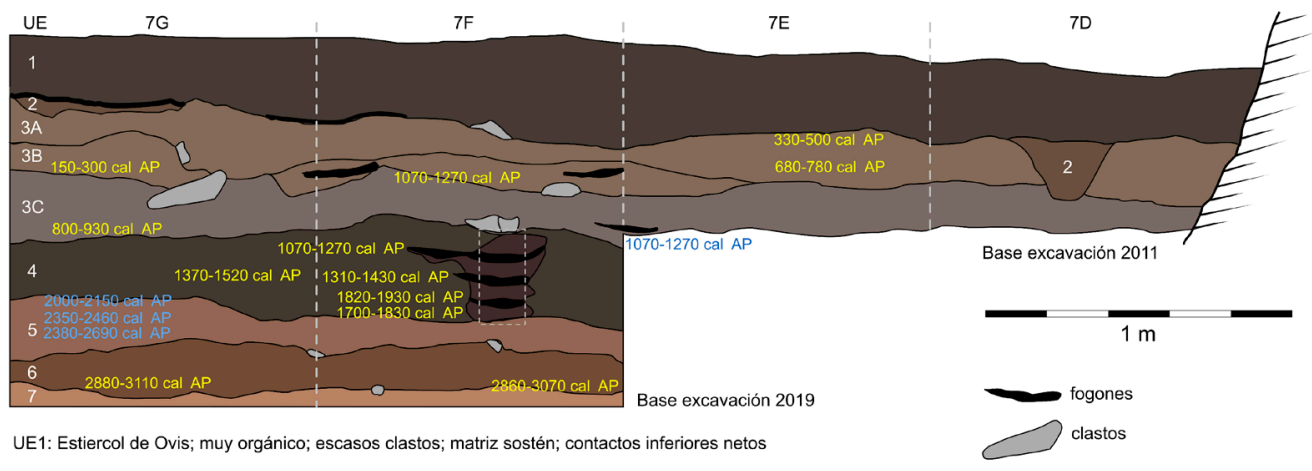

UE2: Arcilla limosa; orgánica; sin clastos; contactos inferiores netos

UE3A: Clastos de meteorización in situ; sin posición preferente; clasto sostén; contactos inferiores difusos

UE3B: Grava y gravilla; inorgánica; abundantes clastos; matriz sostén; contactos inferiores difusos

UE3C: Clastos de meteorización in situ; horizontales a inclinados; clasto sostén; contactos inferiores netos y difusos

UE4: Limo arcilloso; orgánico; abundantes carbones; escasos clastos; matriz sostén; contactos inferiores netos

UE5: Arcilla; abundantes clastos pequeños; matriz sostén grada lateralmente a clasto sostén; contactos inferiores netos y difusos

UE6: Limo arcilloso; orgánico-carbonoso; escasos clastos; matriz sostén; contactos inferiores difusos

UE7: Arena y gravilla; inorgánica; escasos clastos; contacto inferior desconocido

Figura 6. Perfil estratigráfico sur del sitio Cueva Los Carneros. UE: unidad estratigráfica; edades en amarillo provienen de las cuadrículas en las que aparecen; edades en azul están proyectadas de acuerdo a profundidad y UE.

South stratigraphic section of Los Carneros Cave site. UE: stratigraphic unit; ages in yellow are from the quadrats in which they appear; ages in blue are projected according to depth and UE.

de forma continua la UE3 compuesta de clastos de tamaño variable cuya orientación, sostén y presencia de partículas menores permiten definir tres subunidades con un espesor de $25-50 \mathrm{~cm}$. Subyacen tres unidades de limos arcillosos y arcillas de variable contenido orgánico, con distinta proporción de clastos, dominando la matriz como sostén (exceptuando el sector norte de UE5) y con contactos difusos y netos. Infrayace una UE de espesor desconocido (por la interrupción de las excavaciones), compuesta de arenas, gravillas y gravas con bajo contenido orgánico. Los materiales arqueológicos se disponen desde el techo de la UE3 hasta la base excavada, con mayor representación en la UE4. Dieciséis fechados posicionan las ocupaciones del sitio entre 150-300 y 2880-3110 cal. AP. Estas representan 11 eventos ocupacionales (150-300 cal. AP, 330-500 cal. AP, 680-780 cal. AP, 800-930 cal. AP, 1090-1260 cal. AP, 1350-1430 cal. AP, 1700$1830 \mathrm{cal}$. AP, $1820-1930 \mathrm{cal}$. AP, 2000-2150 cal. AP, 2350-2700 cal. AP, 2880-3070 cal. AP), considerando también una edad obtenida en la cámara oeste (Prieto y Mena 2016).

Los materiales corresponden a restos óseos en abundancia, material lítico y carbón, los que se encuentran asociados a 15 rasgos pertenecientes principalmente a fogones, los que se registraron ocasionalmente superpuestos. Dado que el material se encuentra aún en estudio, una cuantificación preliminar de los restos relevados en planta incluye 487 elementos óseos, 153 piezas líticas y 40 fragmentos de carbón. Entre los restos óseos domina Ovis aries en UE1 y Lama guanicoe en UE3, lo que se suma a Hippocamelus bisulcus, carnívoros, dasipódidos, Rhea pennata, aves, y roedores (Mena y López 2014), diversidad que concuerda con lo registrado en las UE inferiores. Los materiales líticos incluyen principalmente variedades de sílices, obsidiana negra, andesita y dacita; las que se usaron para confeccionar localmente una diversidad de herramientas entre las que se incluyen raspadores, raederas, puntas de proyectil, buriles, cuchillos bifaciales y bolas (Figura 7). Adicionalmente, se registraron silicofitolitos afines a Poaceae y Cyperaceae en el residuo de uso de una mano de moler que se registró en un nivel asociado a una edad de 1700-1830 cal. AP.

Alero Entrada Baker (RCH02; $47^{\circ} 12^{\prime} 0.5^{\prime \prime} \mathrm{S}$; 71 54 ' 14.7' W; $635 \mathrm{msm}$ ), ubicado a $4 \mathrm{~km}$ al sur de Cueva Los Carneros, corresponde a un amplio alero donde se condujeron varias campañas de excavación tanto en su interior (Figura 3C) como en su talud (Mena y Jackson 1991; Méndez y Velásquez 2005). Ricos conjuntos de fauna y material lítico caracterizan 

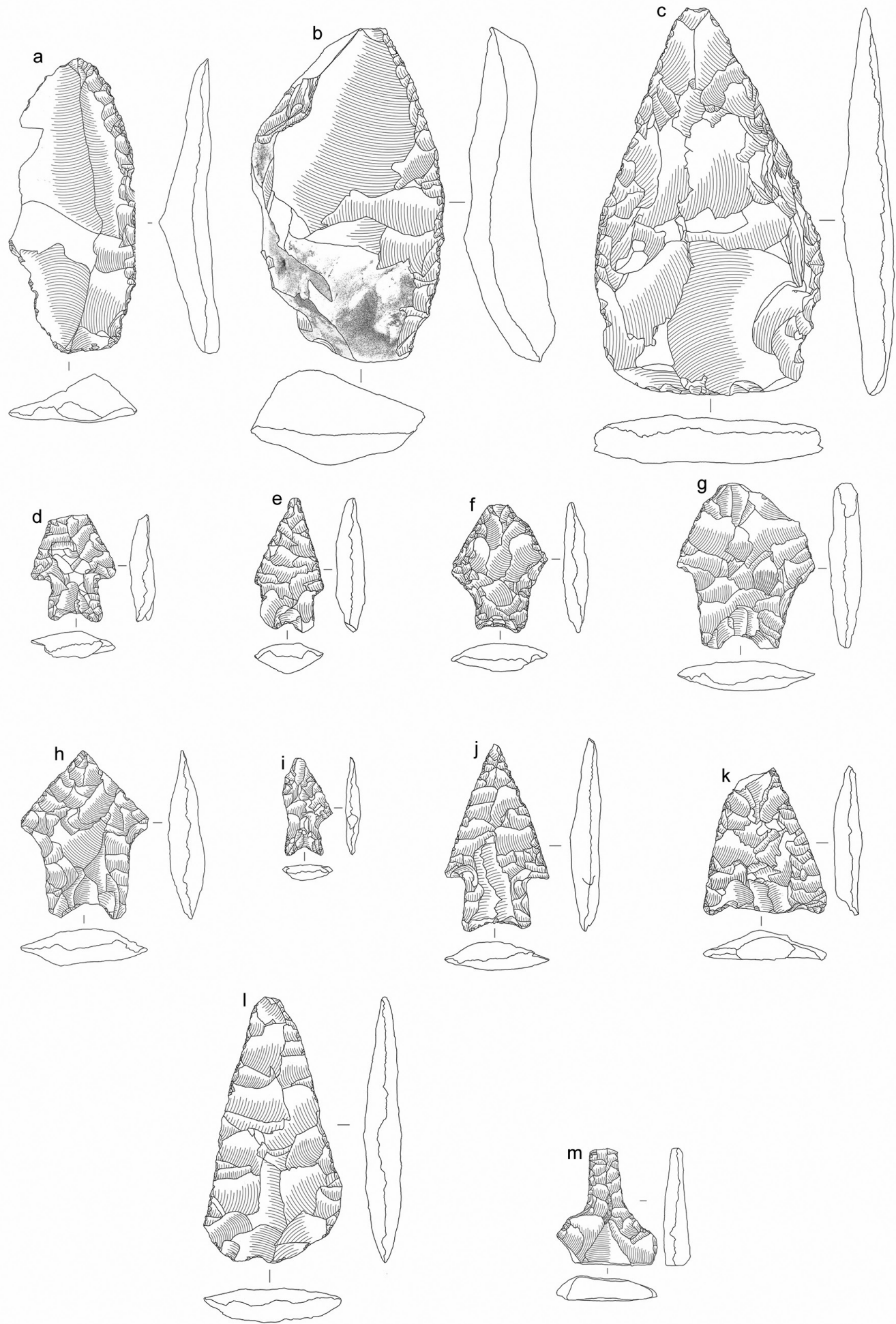

Figura 7. Herramientas líticas seleccionadas de sitios de la cuenca de Chacabuco: raederas (a-c); puntas de proyectil (d-k); cuchillo bifacial (1); lezna/perforador (m). Procedencia: Cueva Los Carneros (a-b, d-f, j-1), Alero Entrada Baker (c, g, i, m) y Alero Gianella (h). Selected lithic tools from sites in the Chacabuco Basin: side scrapers (a-c); projectile points (d-k); bifacial knife (l); awl (m). Origin: Cueva Los Carneros ( $a-b, d-f, j-l)$, Alero Entrada Baker (c, g, $i, m)$, and Alero Gianella $(h)$. 
este sitio que fue interpretado como un campamento transitorio para ocupaciones logísticas (Mena y Jackson 1991). A pesar de las similitudes entre el exterior e interior (i.e., materias primas seleccionadas, diversidad de taxa), los conjuntos líticos sugerían mayor expeditividad en el primero, mientras que la diversidad de fauna (incluyendo huemul y pudú - Pudu pudu) apuntaba a rangos de acción más amplios en el segundo (Méndez y Velásquez 2005). Al igual que en el caso de Los Carneros, una mano de moler registrada en excavación al interior del alero arrojó restos de silicofitolitos afines a Poaceae y Cyperaceae. La escasa precisión en la procedencia de las muestras e inversiones estratigráficas hace difícil entender la secuencia depositacional al interior del reparo, la que se extiende discontinuamente entre 1-390 y 83108780 cal. AP. Por su parte, excavaciones en el talud del alero han demostrado una relación estratigráfica ordenada en el rango entre 300-450 y 4860-5220 cal. AP. Considerando las 10 edades disponibles para este sitio, ha sido posible definir siete eventos ocupacionales cronológicamente independientes (1-390 cal. AP, 310-450 cal. AP, 1070-1240 cal. AP, 1930-2120 cal. AP, $2430-2760$ cal. AP, 4860-5220 cal. AP, 8310-8780 cal. AP).

Prospecciones llevadas a cabo en el año 2000 en el sector de Alto Chacabuco permitieron el reconocimiento de 94 concentraciones y hallazgos aislados de superficie, especialmente al sur del río (Méndez et al. 2004). Luego de la relocalización de uno de ellos, el sitio Laguna Alta (10Sur22C; $47^{\circ} 12^{\prime} 50.9^{\prime \prime} \mathrm{S} ; 7^{\circ} 54^{\prime} 43.8^{\prime \prime} \mathrm{W} ; 648 \mathrm{msm}$ ) fue seleccionado para una excavación de $1 \mathrm{~m}^{2}$ a fin de caracterizar y fechar las ocupaciones a cielo abierto (Figura 4D). Corresponde a una dispersión superficial de material lítico y óseo que se expone por procesos erosivos en el margen de una laguna estacional. La unidad de excavación mostró que a lo largo de $35 \mathrm{~cm}$ se desarrollan dos UE superiores de limos arcillosos con presencia de horizontes de suelo bien definidos que suprayacen a una tercera unidad de arcillas inorgánicas. Los materiales arqueológicos se concentran en la UE intermedia. Dos fechas ${ }^{14} \mathrm{C}$, 660-730 cal. AP en carbón de la excavación y 21502320 cal. AP en colágeno de una muestra de hueso de superficie, proveen edades estadísticamente diferentes para este contexto.

Otros dos reparos rocosos han sido trabajados y fechados en la cuenca del Río Chacabuco. Alero Gianella (RCH01; 47²'47.97” S; 72 ${ }^{\circ} 16^{\prime} 20.31^{\prime \prime} \mathrm{W}$; $686 \mathrm{msm}$ ) corresponde a un reparo con arte rupestre en el que excavaciones $\left(8 \mathrm{~m}^{2}\right)$ relevaron escaso material lítico con dominio de materias primas locales y restos óseos de Lama guanicoe e Hippocamelus bisulcus en asociación a rasgos carbonosos (Fuentes-Mucherl et al. 2012). Dado que se contaba solo con una edad para este depósito, se llevaron a cabo tres nuevos fechados que permiten definir cuatro eventos ocupacionales independientes (450-520 cal. AP, 2180-2380 cal. AP, 2720-2880 cal. AP, 3850-4090 cal. AP). Similar situación constituye el sitio Alero Blanco ( $\mathrm{RCH07}$; de coordenadas desconocidas) que corresponde a un pequeño reparo al este de Alero Gianella (Mena y Blanco 2017). Al igual que en el caso anterior, se contaba con una única edad para este contexto, por lo que dos nuevos análisis permiten definir tres eventos de ocupación humana (340-510 cal. AP, 960-1060 cal. AP, 1070-1260 cal. AP).

\section{Discusión}

Entre los múltiples condicionantes externos que influyen en la ocupación humana de un espacio se encuentran las barreras geográficas, tanto climáticas como geomorfológicas, las que actúan en distintas escalas de tiempo, pudiendo ser temporarias y/o continuas (Veth 1993). En Patagonia, una perspectiva biogeográfica ha sido aplicada para abordar el estudio de las fluctuaciones en la ocupación humana de los espacios y el rol que jugaron las barreras a lo largo del tiempo (Barberena 2008; Borrero 1989-90; Méndez y Reyes 2008; Morello et al. 2012). Por lo tanto, cada espacio puede clasificarse cualitativa y cuantitativamente a través del registro arqueológico según las barreras que presente para los grupos cazadores-recolectores en cada unidad de tiempo (Borrero y Borrazzo 2011). En el occidente de Patagonia, la ocupación humana ha estado condicionada por múltiples barreras biogeográficas, formando espacios denominados Andean dead ends o callejones sin salida de los Andes (Borrero 2004). Este constructo ha sido crucial para la comprensión de las ocupaciones humanas en un área dominada por una geografía que encauzó la distribución de la población a lo largo del Holoceno.

En el oeste de Patagonia, una de las principales barreras corresponde a las grandes masas de hielo que cubrieron la Cordillera de los Andes hasta el Pleistoceno Final y que hoy se ven fundamentalmente reducidas a los campos de Hielo Norte y Sur (Davies et al. 2020). Ríos, lagos y cordones montañosos han sido considerados como factores influyentes en la 
circulación humana y en la distribución de rasgos discretos de la variabilidad tecno-estilística (Borrero y Borrazzo 2011; Franco 2008). Otra barrera influyente fueron los densos bosques que se desarrollaron en la zona abandonada por los glaciares, ocupando sectores con topografía abrupta (Bellelli et al. 2018; Méndez y Reyes 2008; Moreno et al. 2019). Los trabajos comparativos al sur de los $42^{\circ} \mathrm{S}$ concuerdan en que la señal humana en el occidente continental es menor a aquella medida en espacios abiertos contiguos (Belardi y Carballo Marina 2014; Borrero y Muñoz 1999; Reyes et al. 2009; Scheinsohn et al. 2009). Las poblaciones humanas incrementaron su presencia en estos sectores boscosos durante algunos periodos, aunque esto fue principalmente de forma complementaria a la permanencia más sostenida en la estepa (Bellelli et al. 2000; Fernández et al. 2013; Mena 2013; Méndez y Reyes 2008).

El área al este del Campo de Hielo Norte corresponde a uno de los callejones sin salida más claros del oeste de Patagonia y menos estudiados arqueológicamente. Pese a lo limitado que pueden resultar los datos iniciales presentados en este trabajo, el conjunto cronológico obtenido $\left(\mathrm{N}=49\right.$ edades $\left.{ }^{14} \mathrm{C}\right)$ se usó para medir preliminarmente la intensidad de ocupación humana en este espacio (Figura 8). Para ello, los eventos ocupacionales distinguidos a escala de sitio $(\mathrm{N}=38)$ han sido divididos en tres sectores geográficos, potencialmente ejes articuladores de la movilidad. La costa sur del Lago General Carrera muestra una cronología que se inicia hacia $3550 \mathrm{cal}$. AP y se extiende hasta $370 \mathrm{cal}$. AP. Combinados los sitios y depósitos arqueológicos datados, se contabilizan seis eventos ocupacionales, solo observándose redundancia en el sitio Chile Chico 1. Esta zona cuenta con una amplia variabilidad en el registro arqueológico, la que incluye sitios en cuevas, sitios a cielo abierto estratificados, arte rupestre y entierros humanos. Los restos humanos han sido registrados en chenques y muestran una cronología entre 540 y 620 cal. AP, lo que se encuentra de acuerdo con las fechas para este tipo de inhumaciones en la región y áreas aledañas (García Guraieb et al. 2015; Méndez et al. 2017; Reyes 2002). La cerámica registrada, aunque muy escasa a nivel regional, resulta indicativa de procesos de adquisición de una tecnología vinculada a la preparación, consumo y transporte de alimentos. Su cronología por asociación, 370 a $420 \mathrm{cal}$. AP, coincide con los rangos propuestos para la ocupación con cerámica de Alero Entrada Baker y posdata a los fechados directos en este material del sitio Appeleg 1 en el Río Cisnes (Mena y Jackson 1991; Velásquez et al. 2007). En cuanto al arte rupestre, destaca un estilo dominante como son los negativos de mano en rojo, que se encuentran concentrados en el sitio La Tina.

A lo largo del eje del Río Jeinemeni, solo un sitio ha sido fechado, Quebrada Honda. Sin embargo, por sí solos, sus depósitos cubren una cronología entre 500 y $1560 \mathrm{cal}$. AP conformada por cinco eventos ocupacionales en orden estratigráfico, asociados a material arqueológico lítico, óseo y carbón. La presencia adicional de arte rupestre en la Cueva de las Manos del Río Pedregoso, con variados estilos y una alta frecuencia de superposiciones, no solo sugiere una importante redundancia de uso de este espacio, sino una posible presencia temprana sobre la base de algunos de los motivos registrados (Carden y Miotti 2020).

A lo largo del Río Chacabuco se cuenta con una mayor intensidad de trabajo acumulado (Mena y Blanco 2017; Mena y Lucero 2004; Méndez et al. 2004). Su cronología actual se extiende entre 8550 y 200 cal. AP y representa 27 eventos ocupacionales. La alta frecuencia de estos está dada por el dominio de sitios en cuevas, los que son contextos recurrentemente ocupados y que promueven la supervivencia del material fechable (Strauss 1991). Las edades de termoluminiscencia disponibles para seis sitios del valle solo cubren parcialmente la distribución de ${ }^{14} \mathrm{C}$, ya que se extienden entre 2200 y $1000 \mathrm{AP}(200$ AC - 1000 DC; Mena et al. 2014). Los materiales registrados en estos sitios son muy abundantes y muestran una importante diversidad de rocas de alta calidad para la talla, las que se observan en conjuntos formatizados (Méndez, Stern et al. 2018). Los restos de fauna muestran un aprovechamiento de la diversidad de especies disponibles a nivel del valle, incluyéndose cérvidos, posiblemente transportados hasta la estepa (Méndez y Velásquez 2005). El arte rupestre se caracteriza por ser escaso (i.e., Alero Gianella, Alero Los Niños y Alero Entrada Baker) y está representado por pocos motivos por cada sitio (Fuentes Mucherl et al. 2012; Mena y Blanco 2017).

La información arqueológica recabada en los valles andinos de Patagonia Centro Occidental es disímil. Entre los $44^{\circ}$ y $45^{\circ} 30^{\prime} \mathrm{S}$, los datos cronológicos sugieren una señal humana intermitente distribuida a lo largo de todo el Holoceno (Méndez, Nuevo Delaunay et al. 2018). Sitios bajo reparo rocoso como El Chueco 1, Cueva de la Vieja y Baño Nuevo 1, ubicados en las 


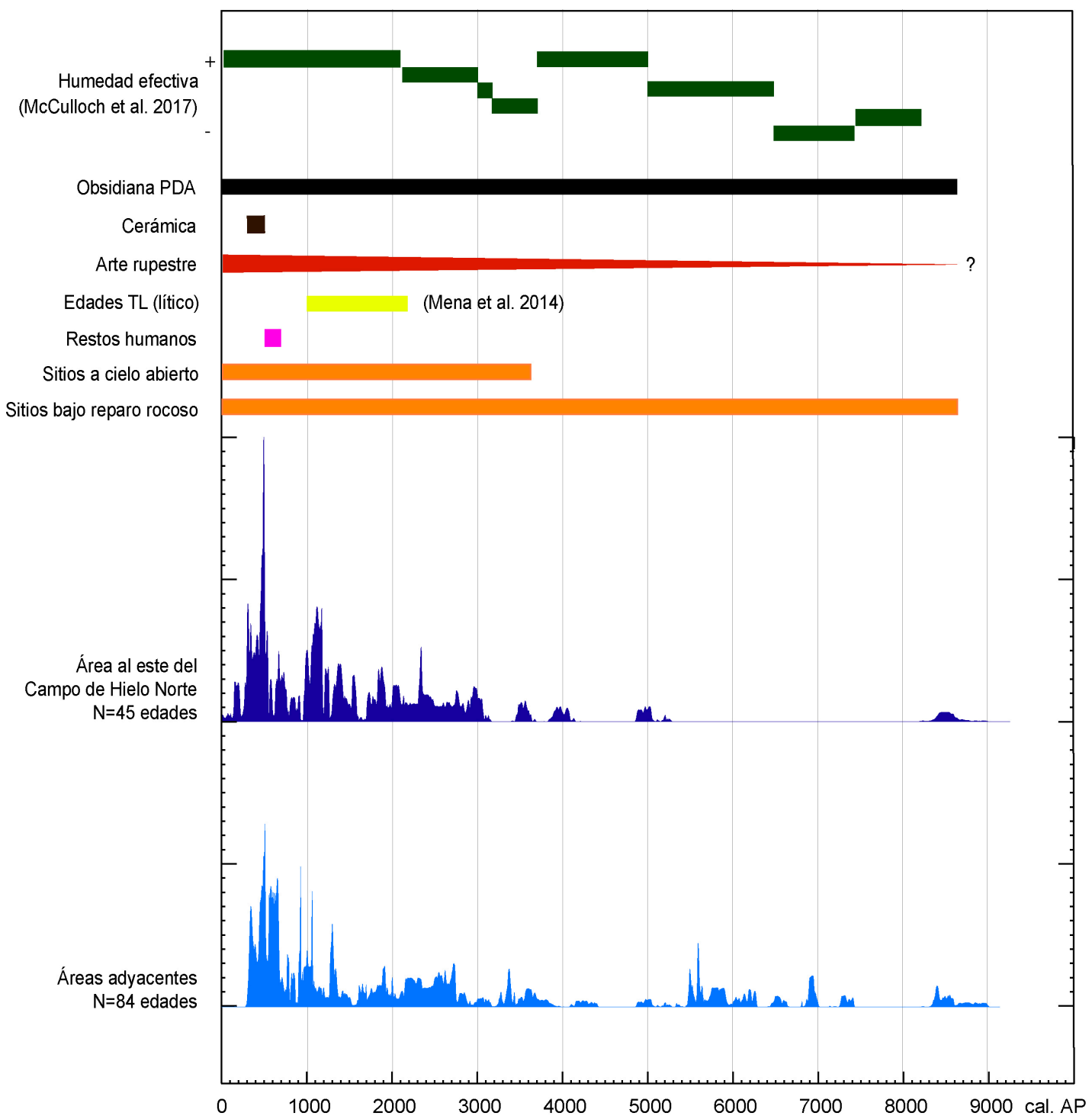

Figura 8. Cronología del área al este del Campo de Hielo Norte y áreas adyacentes (distribución de suma de probabilidades), indicadores arqueológicos y humedad efectiva (escala relativa aproximada).

Chronology of the area east of the Northern Icefield and adjacent areas (summed probability distributions), archaeological indicators, and effective moisture (approximate relative scale).

estepas orientales de Cisnes y Nirehuao, dan cuenta de secuencias depositacionales discontinuas desde $\sim 12000$ cal. AP hasta ocupaciones modernas (Mena y Stafford 2006; Méndez, De Porras et al. 2016; Méndez, Stern et al. 2018). Al este del Campo de Hielo Norte, no hay aún ningún sitio que por sí solo muestre este grado de redundancia ocupacional, como tampoco lo muestran los datos acumulados a escala de cuenca. Durante el Holoceno Temprano/Medio, a las secuencias de Cisnes y Ñirehuao se suman nuevos sitios en estos valles y otros en Coyhaique Alto y en el Río Ibáñez, más cercanos a los bosques o dentro de ellos, donde se detectaron nuevas ocupaciones de carácter más puntual (Mena 2000; Méndez, Reyes et al. 2016; Nuevo Delaunay et al. 2013). Si bien es cierto el sitio Alero Entrada Baker muestra presencia humana durante este periodo $(8310-8780 \mathrm{cal}$. AP), la evidencia aún es insuficiente para afirmar una continuidad en la ocupación del área de estudio. A partir de los 3000 cal. AP, todos los sectores de 
Aisén continental muestran ocupaciones, las cuales se manifiestan no solo en reparos rocosos, sino también a cielo abierto y en una multiplicidad de funciones que incluyen sitios habitacionales, canteras-talleres, entierros humanos y ocupaciones efímeras en variados ambientes (Gómez y Méndez 2015; Méndez y NuevoDelaunay 2019; Méndez et al. 2017; Reyes 2002; Thompson y Méndez 2019). La ocupación al este del Campo de Hielo Norte se concentra en este periodo y se vuelve continua (Figura 8). Sitios de todos estos tipos han sido detectados en cada una de las subáreas muestreadas y eventos ocupacionales redundantes son relativamente frecuentes a escala de sitio (p.ej., Cueva Los Carneros, Quebrada Honda).

Algunos indicadores arqueológicos permiten comparar decisiones humanas en escala regional en términos de sus continuidades y discontinuidades (Figura 8). Por ejemplo, la distribución de la obsidiana, trazada geoquímicamente, corresponde a un elemento exótico común a todas las ocupaciones continentales de Patagonia Central (Stern 2018). A lo largo del Holoceno dominó la obsidiana procedente de Pampa del Asador, la que se comporta con un decrecimiento exponencial de sur a norte en función de la distancia a la fuente, tanto a escala de sitios, como de cuencas (Santa Cruz, Argentina) y solo con una mayor variabilidad al norte de los $45^{\circ} \mathrm{S}$ (Méndez, Stern et al. 2018). El arte rupestre, por su parte, solo es muy frecuente al norte y sur del Lago General Carrera, ya que su presencia decrece en la medida que uno se aleja de estos sectores (Cordero et al. 2019; Fuentes-Mucherl et al. 2012; Méndez, Reyes et al. 2016; Nuevo Delaunay et al. 2013). Una distribución similar se observa con el patrón funerario de chenques, los cuales son frecuentes en torno al lago y muy escasos lejos de este (Goñi et al. 2004; Méndez et al. 2017; Reyes 2002; Reyes y Méndez 2010). Por su parte, la cerámica es un elemento tardío de muy baja presencia en cada uno de estos valles, la que sin embargo ha podido ser detectada en Cisnes, Ibáñez, Jeinemeni y Chacabuco (Mena y Jackson 1991; Mena y Lucero 2004; Velásquez et al. 2007).

Finalmente, los sectores extraandinos inmediatamente al este del Campo de Hielo Norte demuestran algunos paralelos importantes a nuestra área de estudio, especialmente en cuanto a la distribución cronológica (Figura 8). Los trabajos desarrollados en la costa noreste del Lago Pueyrredón muestran una alta densidad arqueológica, con sitios bajo reparo rocoso y a cielo abierto, y en donde las intervenciones estratigráficas realizadas en Milodón Norte 1 y Cerro Cuadrado 3 han arrojado edades iniciales de 8630-8990 cal. AP y 8320-8460 cal. AP, respectivamente (Aschero et al. 2009; Sacchi et al. 2016). Estas fechas traslapan con la primera edad registrada en Alero Entrada Baker, distante a menos de $12 \mathrm{~km}$ hacia el norte (Mena y Jackson 1991). Por otro lado, los trabajos desarrollados entre el Lago Pueyrredón y el Lago Ghio dan cuenta de ocupaciones discontinuas entre 7500 y 2800 años cal. AP, principalmente en contextos bajo reparo rocoso; tanto en los mencionados sitios como en Estancia Pueyrredón 1 y 2, Sol de Mayo I Colmillo Sur I, con alta redundancia ocupacional (Figuerero Torres y Mengoni Goñalons 2006; Mengoni Goñalons et al. 2019; Sacchi et al. 2016). Durante este periodo, los grupos humanos enfrentaron importantes cambios en la morfología y tamaño de los lagos, cuestión que afectó la circulación a través de la región (Horta et al. 2019). En términos ambientales, después de $6500 \mathrm{cal}$. AP, se observan condiciones regionales de mayor humedad (posiblemente más cálidas) que, aunque fluctuaron mucho, permitieron el desarrollo de un bosque en altura $(1.000 \mathrm{msm})$, como lo sugiere el perfil sedimentario de La Frontera (McCulloch et al. 2017). La mayor presencia humana en toda el área, incluyendo las cuencas de los lagos Posadas y Salitroso, se evidencia a partir de los últimos tres milenios, como lo sugiere la continuidad en las distribuciones radiocarbónicas durante este lapso temporal (García Guraieb et al. 2015; Goñi et al. 2019; Mengoni Goñalons et al. 2019). No hay que descontar la influencia de la tafonomía del material orgánico fechable, ya que su preservación diferencial podría estar influenciando la mayor representación de registros arqueológicos más recientes. No obstante, esta mayor densidad del registro radiocarbónico se ve aparejada con un incremento en los episodios de fuego, en los cuales posiblemente hubo influencia humana como se ha detectado en otras partes de la región (McCulloch et al. 2017; Méndez, De Porras et al. 2016).

\section{Conclusiones}

El concepto de Andean dead ends ha resultado clave en la comprensión del uso humano del oeste de Patagonia porque incorpora variables geográficas en el estudio arqueológico, abordando cómo las condicionantes del espacio encausaron la distribución de los grupos de cazadores recolectores durante el Holoceno (Borrero 2004). Nuestra evaluación cualitativa y cuantitativa del este del Campo de Hielo Norte buscó, a través de indicadores como la distribución de sitios, su cronología y redundancia ocupacional, entender las trayectorias arqueológicas y su variabilidad en distintas escalas espaciales dentro de 
un espacio relativamente circunscrito. Combinando la información del área de estudio y la de zonas adyacentes, observamos una primera etapa de ocupaciones discontinuas que preliminarmente se inician hace 8600 años y que se extienden hasta hace 3000 años atrás. En esta fecha se desarrolló una mayor cantidad de ocupaciones que se observan en distintos sectores, con registros redundantes, no solo en cuevas, sino en localidades a cielo abierto, lo que incorpora una nueva fuente de información cronológica. Aparejado a esta mayor densidad en la distribución radiocarbónica, se observa mayor variabilidad en los contextos y conjuntos, lo que sugiere una mayor continuidad en las ocupaciones.

La información recuperada en esta área, aunque preliminar, posee importantes perspectivas para la investigación regional futura. Como corredores, resulta indispensable abordar la variabilidad en el sentido este-oeste que permite ejes espaciales como la costa sur del Lago General Carrera o el Río Chacabuco. Ello permitirá evaluar si áreas más cerradas como los bosques occidentales poseen una cronología diferente a las de áreas abiertas, o si el Río Baker y el Campo de Hielo Norte constituyeron verdaderas barreras que inhibieron el desplazamiento humano.

\section{Referencias Citadas}

Aschero, C., D. Bozzuto, M.T. Civalero, M. De Nigris, A. Di Vruno, V. Dolce, N. Fernández, L. González y P. Limbrunner 2009. El registro arqueológico de la costa noreste del Lago Pueyrredón-Cochrane (Santa Cruz, Argentina). En Arqueología de Patagonia: Una Mirada desde el Último Confín, editado por M. Salemme, F. Santiago, M. Álvarez, E. Piana, M. Vázquez y M.E. Mansur, pp. 919-926. Editorial Utopías, Ushuaia.

Aschero, C., R. Goñi, M.T. Civalero, R. Molinari, S. Espinosa, A.G. Guraieb y C. Bellelli 2005. Holocenic Park: Arqueología del Parque Nacional Perito Moreno (PNPM). Anales de Parques Nacionales XVII:71-119.

Barberena, R. 2008. Arqueología y Biogeografía Humana en Patagonia Meridional. Sociedad Argentina de Antropología, Buenos Aires.

Behrensmeyer, A.K. 1978. Taphonomic and ecological information from bone weathering. Paleobiology 4 (2):150-162.

Belardi, J.B. y F. Carballo Marina 2014. La señal arqueológica en el interior del bosque en la margen sur del lago San Martín (provincia de Santa Cruz). Comechingonia 18 (2):181-202.

Bellelli, C., M. Carballido Catalayud y C.R. Stern 2018. Obsidianas en el bosque: determinación geoquímica de artefactos arqueológicos del S-O de Río Negro y N-O de Chubut (Patagonia Argentina). Chungara Revista de Antropología Chilena 50 (2):201-216.

Bellelli, C., V. Scheinsohn, P. Fernández, F. Pereyra, M.M. Podestá y M. Carballido 2000. Arqueología de la Comarca Andina del Paralelo 42. Localidad de Cholila. Primeros resultados. En Desde el País de los Gigantes: Perspectivas Arqueológicas en
Agradecimientos: Un agradecimiento muy especial a Luis Borrero, maestro y amigo, por su constante apoyo y guía. Financiado por los proyectos ANIDFONDECYT \#1180306, \#1210042, \#11180388, \#1210045 programa ANID Regional \#R20F0002, FIC BIP \#40000234-0 y National Geographic Grant \#HJ-150R-17. Agradecemos a Dagoberto Guzmán, Christian Saucedo, Cristian Saldia, Mauricio Quercia, Sergio Haro, Sergio Giménez, Carlos Maglio, Marcelo Herrera, Ceferino e Ignacio Márquez y IV División del Ejército de Chile, Carabineros de Chile, Tompkins Conservation y IV División de Ejército de Chile, Carabineros de Chile, Tompkins Conservation y Parque Nacional Patagonia (CONAF) por los permisos y apoyo en nuestros trabajos; a Valentina Trejo, Damián Bozzuto, Patricio López, Carola Flores, Paulo Moreno, Bárbara Thompson, Javier Carranza, Nicolás Araneda, Pedro Fuentes, Joaquín Crisóstomo, Constanza Neira, Antonia Fuenzalida, Francisca Moya, Rosario Cordero, Ivonne Farías, Gustavo Fredes, por la ayuda en terreno y laboratorio; a Robert McCulloch, por la ayuda con la interpretación ambiental. Agradecemos los comentarios de dos evaluadores anónimos que ayudaron a mejorar el artículo.

Patagonia, editado por J.B. Belardi, F. Carballo y S. Espinosa, tomo II, pp. 587-602. Universidad Nacional de la Patagonia Austral, Río Gallegos.

Belmar, C. 2019. Los Cazadores-Recolectores y las Plantas en Patagonia. Perspectivas desde el Sitio Cueva Baño Nuevo 1, Aisén. Social Ediciones, Santiago.

Belmar, C., C. Méndez y O. Reyes 2017. Hunter-gatherer plant resource use during the Holocene in central western Patagonia (Aisén, Chile, South America). Vegetation History and Archaeobotany 26 (6):607-625.

Borrero, L.A. 1989-90. Evolución cultural divergente en la Patagonia austral. Anales del Instituto de la Patagonia (serie Ciencias Humanas) 8:81-93.

Borrero, L.A. 2004. The archaeozoology of the Andean 'Dead Ends' in Patagonia: Living near the continental ice cap. En Colonisation, Migration, and Marginal Areas: A Zooarchaeological Approach, editado por M. Mondini, S. Muñoz y S. Wickler, pp. 55-61. Oxbow Books, Havertown.

Borrero, L.A. y K. Borrazzo 2011. Bosques, Montañas y Cazadores: Investigaciones Arqueológicas en Patagonia Meridional. Editorial Dunken, Buenos Aires.

Borrero, L.A. y S. Muñoz 1999. Tafonomía en el bosque patagónico. Implicaciones para el estudio de su explotación y uso por poblaciones humanas de cazadores-recolectores. En Soplando en el Viento, editado por J.B Belardi, P. Fernández, R. Goñi, A. G. Guráieb y M. de Nigris, pp. 43-56. INAPL, Buenos Aires. 
Borrero, L.A., A. Nuevo Delaunay y C. Méndez 2019. Ethnographical and historical accounts for understanding the exploration of new lands: The case of Central Western Patagonia, Southernmost South America. Journal of Anthropological Archaeology 54:1-16.

Carballo, F., J.B. Belardi, y L.A. Borrero 2016. Nuevos datos para la discusión arqueológica de corredores en el bosque del suroeste de la provincia de Santa Cruz, Argentina: el caso del arroyo Los Loros. Magallania 44 (2):209-217.

Carden, N. y L. Miotti 2020. Unraveling rock art palimpsests through superimpositions: The definition of painting episodes in Los Toldos (southern Patagonia) as a baseline for chronology. Journal of Archaeological Science: Reports 30:102265.

Coil, J., A. Korstanje, S. Archer y C. Hastorf 2003. Laboratory goals and considerations for multiple microfossil extraction in archaeology. Journal of Archaeological Science 30:991-1008.

Contreras, C. 2012. Tecnología lítica en la cuenca del río Jeinemeni: análisis de material superficial. Boletín de la Sociedad Chilena de Arqueología 41-42:29-42.

Cordero, R., C. Muñoz y D. Artigas 2019. Reinterpretando paredes: Interacción e intercambio de información en el Ibáñez medio, Patagonia central, Chile. Boletín del Museo Chileno de Arte Precolombino 24 (1):37-55.

Davies, B.J., C.M. Darvill, H. Lovell, J.M. Bendle, J.A. Dowdeswell, D. Fabel, J-L. García, A. Geiger, N.F. Glasser, D.M. Gheorghiu, S. Harrison, A.S. Hein, M.R. Kaplan, J.R.V. Martin, M. Mendelova, A. Palmer, M. Pelto, Á. Rodés, E.A. Sagredo, R. Smedley, J.L. Smellie y V.R. Thorndycraft 2020. The evolution of the Patagonian Ice Sheet from $35 \mathrm{ka}$ to the Present Day (PATICE). Earth-Science Reviews 204:103152.

De Porras, M.E., A. Maldonado, A.M. Abarzúa, M.L. Cárdenas, J.P. Francois, A. Martel-Cea, C.R. Stern, C. Méndez y O. Reyes 2012. Postglacial vegetation, fire y climate dynamics at Central Chilean Patagonia (Lake Shaman, $44^{\circ} \mathrm{S}$ ). Quaternary Science Reviews 50:71-85.

De Porras, M.E., A. Maldonado, F. A. Quintana, A. Martel-Cea, O. Reyes, y C. Méndez 2014. Environmental and climatic changes in central Chilean Patagonia since the Late Glacial (Mallín El Embudo, $44^{\circ}$ S). Climate of the Past 10 (3):1063-1078.

Ericksen, M.F. 1965. Restos óseos provenientes de Chile Chico (provincia de Aisén, Chile). Revista Universitaria 2:50-51.

Fernández, P.M., M. Carballido, C. Bellelli y M. Podestá 2013. Tiempo de cazadores. Cronología de las ocupaciones humanas en el valle del río Manso inferior (Río Negro). En Tendencias Teórico-Metodológicas y Casos de Estudio en la Arqueología de Patagonia, editado por A.F. Zangrando, R. Barberena, A. Gil, G. Neme, M. Giardina, L. Luna, C. Otaola, S. Paulides, L.Salgán y A.Tivoli, pp.167-175. Editorial Altuna, Buenos Aires.

Figuerero Torres, M.J. y G.L. Mengoni Goñalons 2006. Lugares persistentes y vacíos ocupacionales en sociedades cazadorasrecolectoras de la Patagonia centro-meridional. Actas del XVII Congreso Nacional de Arqueología Chilena, pp. 1319-1330. Ediciones Kultrún, Valdivia.

Franco, N. 2008. La estructura tecnológica regional y la comprensión de la movilidad humana: Tendencias para la cuenca del río Santa Cruz. En Arqueología del Extremo Sur del
Continente Americano, editado por L.A. Borrero, pp. 119-151. Dunken, Buenos Aires.

Fuentes-Mucherl, F., F. Mena, J. Blanco y C. Contreras 2012. Excavaciones en Alero Gianella, curso medio del valle de Chacabuco (Andes Centropatagónicos). Magallania 40 (2):259-265.

García Guraieb, S., R. Goñi y A. Tessone 2015. Paleodemography of late Holocene hunter-gatherers from Patagonia (Santa Cruz, Argentina): an approach using multiple archaeological and bioarchaeological indicators. Quaternary International 356:147-158.

García, C. y F. Mena 2016. ¿La frontera del oeste? Prospecciones arqueológicas en el bosque montano del extremo occidental del valle medio del río Ibáñez (Andes patagónicos, Chile). Intersecciones en Antropología 17:49-61.

García, J.L., A. Maldonado, M.E. de Porras, A. Nuevo Delaunay, O. Reyes, C.A. Ebensperger, S.A. Binnie, C. Lüthgens y C. Méndez 2019. Early deglaciation and paleolake history of Río Cisnes Glacier, Patagonian Ice Sheet $\left(44^{\circ} \mathrm{S}\right)$. Quaternary Research 91 (1):194-217.

Garreaud, R.D. 2009. The Andes climate and weather. Advances in Geosciences 22:3-11.

Gómez, M.L., y C. Méndez 2015. Aprovisionamiento lítico en Alto Río Cisnes, el caso del sitio La Cantera 1. Actas del XIX Congreso Nacional de Arqueología Chilena, pp. 577-584, Sociedad Chilena de Arqueología y Universidad de Tarapacá, Arica.

Goñi, R., G. Barrientos, M. J. Figuerero, G. Mengoni, F. Mena, V. Lucero y O. Reyes 2004. Distribución espacial de entierros en la cordillera de Patagonia centro-meridional (Lago Salitroso-Paso Roballos, Argentina/Entrada Baker-Chacabuco, Chile). Chungara Revista de Antropología Chilena 36 Número Especial 2:1101-1107.

Goñi R., A. Re, S. García Guraieb, G. Cassiodoro, A. Tessone, D. Rindel, J. Dellepiane, J. Flores Coni, F. Guichón, A. Agnolin 2019. Climate changes, human peopling and regional differentiation during late Holocene in Patagonia. Quaternary International 505:4-20.

Henríquez, W.I., R. Villa-Martínez, I. Vilanova, R. De Pol-Holz y P.I. Moreno 2017. The last glacial termination on the eastern flank of the central Patagonian Andes $\left(47^{\circ} \mathrm{S}\right)$. Climate of the Past 13:879-895.

Hogg, A.G., Q. Hua, P.G. Blackwell, M. Niu, C.E. Buck, T.P. Guilderson, T.J. Heaton, J.G. Palmer, P.J. Reimer, R.W. Reimer, C.S.M. Turney y S.R.H. Zimmerman 2013. SHCal13 Southern Hemisphere calibration, 0-50,000 years cal BP. Radiocarbon 55 (4):1889-1903.

Horta, L.R., M.A. Marcos, M. Sacchi, D. Bozzuto, S.M. Georgieff, M.V. Mancini y M.T. Civalero 2019. Paleogeographic and paleoenvironmental evolution in northwestern Santa Cruz (Argentina), and its influence on human occupation dynamics during the late Pleistocene- early Holocene. Palaeogeography, Palaeoclimatology, Palaeoecology 516:44-53.

ICSN 2011. The International Code for Starch Nomenclature. www.fossilfarm.org/ICSN/Code.html (acceso 2012).

Luebert, F. y P. Pliscoff 2006. Sinopsis Bioclimática y Vegetacional de Chile. Editorial Universitaria, Santiago. 
Madella, M., A. Alexandre y T. Ball 2005. International code for phytolith nomenclature. 1.0. Annals of Botany 96 (2):253-260.

McCulloch, R.D., M.J. Figuerero Torres, G.L. Mengoni Goñalons, R. Barclay y C. Mansilla 2017. A Holocene record of environmental change from Río Zeballos, central Patagonia. The Holocene 27 (7):941-950.

Mena, F. 1987. Visita preliminar sector arqueológico Pampa la Perra. Aonikenk 1 (2):13-16.

Mena, F. 2000. Un panorama de la prehistoria de Aisén oriental: estado del conocimiento a fin de siglo. Serie Antropología 2:21-41.

Mena, F. 2013. ¿Un caso de microidentidad y/o restricción territorial en el Curso Medio del Río Ibáñez? (Aisén, Andes Centropatagónicos, Chile). En Tendencias Teórico-Metodológicas y Casos de Estudio en la Arqueología de la Patagonia, editado por A.F. Zangrando, R. Barberena, A. Gil, G. Neme, M. Giardina, L. Luna, C. Otaola, S. Paulides, L.Salgán y A.Tivoli, pp. 187-192. Editorial Altuna, Buenos Aires.

Mena, F. y J. Blanco 2017. Estado de la investigación arqueológica del valle del Chacabuco. Andes Centro Patagónicos. XI Región de Aisén, Chile. Magallania 45 (2):199-217.

Mena, F., J. Blanco, A. Román y F. Mariani 2014. Thermoluminescence dating of surface lithic artefacts from the Chacabuco Valley, Chilean Patagonia. Archaeometry 56 (5):898-911.

Mena, F. y D. Jackson 1991. Tecnología y subsistencia en el Alero Entrada Baker, Región de Aisén, Chile. Anales del Instituto de la Patagonia. Serie Ciencias Humanas 20:169-203.

Mena, F. y P. López 2014. Los restos faunísticos de los niveles superiores de Cueva Los Carneros (Alto Chacabuco, XI Región, Chile). Revista Chilena de Antropología 30 (2):121-128.

Mena, F. y V. Lucero 2004. En torno a las últimas poblaciones indígenas de la cordillera centro-patagónica: estudio comparado de tres valles en Aisén oriental (Chile). En Contra Viento y Marea. Arqueología de Patagonia, editado por M. Civalero, P. Fernández y G. Guráieb, pp. 643-657. INAPL, Buenos Aires.

Mena, F. y T. Stafford 2006. Contexto estratigráfico y fechación directa de esqueletos humanos del Holoceno temprano en Cueva Baño (Patagonia Central, Chile). En $2^{\circ}$ Simposio Internacional del Hombre Temprano en América, editado por J. Jiménez, S. González, J. Pompa y F. Ortíz, pp. 139-154. INAH, México DF.

Méndez, C. 2004. Movilidad y manejo de recursos líticos de tres valles andinos de Patagonia centro occidental. En Contra Viento y Marea. Arqueología de Patagonia, editado por M. Civalero, P. Fernández y G. Guráieb, pp. 135-147. INAPL, Buenos Aires.

Méndez, C. 2013. Terminal Pleistocene/early Holocene 14C dates form archaeological sites in Chile: Critical chronological issues for the initial peopling of the region. Quaternary International 301:60-73.

Méndez, C., R. Barberena, O. Reyes y A. Nuevo Delaunay 2014. Isotopic ecology and human diets in the forest-steppe ecotone, Aisén Region, Central-Western Patagonia, Chile. International Journal of Osteoarchaeology 24 (2):187-201.

Méndez, C, J. Blanco y C. Quemada 2004. Aprovechamiento de materias primas líticas en el Alto Chacabuco. Chungara Revista de Antropología Chilena 36 Número Especial: 37-48.
Méndez, C., M.E. De Porras, A. Maldonado, O. Reyes, A. Nuevo Delaunay y J.L. García 2016. Human effects in Holocene fire dynamics of Central Western Patagonia ( $44^{\circ} \mathrm{S}$, Chile). Frontiers in Ecology and Evolution 4:100. https://doi.org/10.3389/ fevo.2016.00100

Méndez, C. y A. Nuevo-Delaunay 2019. Evidencias a cielo abierto para discutir superficies potenciales de actividad temprana en Patagonia centro occidental (44-45 $\left.{ }^{\circ} \mathrm{S}\right)$. Magallania 47 (1):105-116.

Méndez, C., A. Nuevo Delaunay, O. Reyes, I.L. Ozán, C. Belmar y P. López 2018. The initial peopling of Central Western Patagonia (southernmost South America): Late Pleistocene through Holocene site context and archaeological assemblages from Cueva de la Vieja site. Quaternary International 473:261-277.

Méndez, C. y O. Reyes 2008. Late Holocene human occupation of the Patagonian forests: a case study in the Cisnes river basin. Antiquity 82 (317):560-570.

Méndez, C., O. Reyes, A. Nuevo Delaunay y E. Latorre 2017. Chenques en el centro oeste de Patagonia (Holoceno tardío final, valle de Nirehuao, $45^{\circ} \mathrm{S}$, Chile). Chungara Revista de Antropología Chilena 49 (3): 379-395.

Méndez, C., O. Reyes, A. Nuevo Delaunay, H. Velásquez, V. Trejo, N. Hormazábal, M. Solari y C.R. Stern 2016. Las Quemas rockshelter: understanding human occupations of Andean forests of Central Patagonia (Aisén, Chile), Southern South America. Latin American Antiquity 27 (2):207-226.

Méndez, C., C.R. Stern, A. Nuevo Delaunay, O. Reyes, F. Gutiérrez y F. Mena 2018. Spatial and temporal distributions of exotic and local obsidians in Central Western Patagonia, southernmost South America. Quaternary International 468:155-168.

Méndez, C. y H. Velásquez 2005. Tecnología y subsistencia en Alero Entrada Baker: una revisión a la luz de nuevos antecedentes. Actas del XVI Congreso Nacional de Arqueología Chilena, pp. 593-602. Sociedad Chilena de Arqueología, Tomé.

Mengoni Goñalons, G.L., M.J. Figuerero Torres, M.V. Fernández, M. Rocco y W. Dekmak 2019. Persistencia y hiatos en el área de Los Antiguos, Monte Zeballos y Paso Roballos, NO de Santa Cruz, Patagonia: nuevas dataciones para la cronología regional. En Arqueología de la Patagonia: el Pasado en las Arenas, editado por J. Gómez Otero, A. Svoboda y A. Banegas, pp. 327-338. Instituto de Diversidad y Evolución Austral, Puerto Madryn.

Morello, F., L.A. Borrero, M. Massone, C.R. Stern, A. GarcíaHerbst, R. McCulloch, M. Arroyo-Kalin, E. Calás, J. Torres, A. Prieto, I. Martínez, G. Bahamonde y P. Cárdenas 2012. Huntergatherers, biogeographic barriers and the development of human settlement in Tierra del Fuego. Antiquity 86 (331):71-87.

Moreno, P.I., E. Simi, R.P. Villa-Martínez e I. Vilanova 2019. Early arboreal colonization, postglacial resilience of deciduous Nothofagus forests, and the Southern Westerly Wind influence in central-east Andean Patagonia. Quaternary Science Reviews 218:61-74

Niemeyer, H. 1978. La cueva con pinturas indígenas del río Pedregoso (Departamento de Chile Chico, Prov. de Aysén, Chile). Notas del Museo de Historia Natural de San Rafael 19:339-353.

Nuevo Delaunay, A., C. Méndez, O. Reyes y V. Trejo 2013. Evaluando evidencias humanas en la margen bosque-estepa 
( $>900$ msnm) de Patagonia central: cueva de Punta del Monte (Región de Aisén, Chile). Magallania 41 (2):127-144.

Pallo, M.C. y L.A. Borrero 2015. Arqueología de corredores boscosos en Patagonia Meridional: el caso del río Guillermo (SO de la provincia de Santa Cruz, Argentina). Intersecciones en Antropología 16:313-326.

Pérez, S.I., M.B. Postillone, D. Rindel, D. Gobbo, P.N. González y V. Bernal 2016. Peopling time, spatial occupation and demography of Late Pleistocene-Holocene human population from Patagonia. Quaternary International 425:214-223.

Prates, L., G. Politis y J. Steele 2013. Radiocarbon chronology of the early human occupation of Argentina. Quaternary International 301:104-122.

Prieto, A. y F. Mena 2016. Dos ganchos de estólica de la estepa patagónica occidental: similares y distantes. Cuadernos del Instituto Nacional de Antropología y Pensamiento Latinoamericano 25 (2):233-236.

Reyes, O. 2002. Funebria Indígena en el curso inferior del valle del río Ibáñez, margen occidental de la estepa Centropatagónica (XI Región de Aisén). Anales del Instituto de Patagonia, Serie Ciencias Sociales 30:87-102.

Reyes, O. 2004. Contextos Funerarios del Holoceno Tardío en el Margen Oriental de la Cordillera de Aisén (XI Región); Curso Inferior de los Valles del Río Ibáñez y Jeinemeni y Curso Superior del Valle del Río Chacabuco. Patagonia Central. Memoria para optar al título de arqueólogo. Departamento de Antropología, Universidad de Chile, Santiago.

Reyes, O. y C. Méndez 2010. Precisando la cronología para la inhumación tipo chenque, valle del río Cisnes (Aisén, Chile), Patagonia Central. Magallania 38 (2):97-102.

Reyes, O., C. Méndez, A. Maldonado, H. Velásquez, V. Trejo, M. Cárdenas y A.M. Abarzúa 2009. Uso del espacio de cazadores recolectores y paleoambiente Holoceno en el valle del río Cisnes, región de Aisén, Chile. Magallania 37 (2):91-107.

Romero, H. 1985. Geografía de los Climas. Geografía de Chile, Tomo XI. Instituto Geográfico Militar, Santiago.

Sacchi, M., D. Bozzuto, L. Horta, N. Fernández, M.E. de Nigris, M.T. Civalero y C.A. Aschero 2016. Dataciones y circulación humana: influencia de las fluctuaciones del sistema lacustre Pueyrredón-Posadas durante el Holoceno. Andes 27:1-16.
Sade, K., F. Castañeda y L. Pérez-Barría 2019. Poblamiento y registro arqueológico de la costa sur del Lago General Carrera (Río Baker, Región de Aysén, Chile). Aysenología 6:29-49.

Scheinsohn, V., C. Szumik, S. Leonardt y F. Rizzo 2009. Distribución espacial del arte rupestre en el bosque y la estepa del norte de Patagonia. Nuevos resultados. En Arqueología de Patagonia: Una Mirada desde el Último Confín, editado por M. Salemme, F. Santiago, M. Álvarez, E. Piana, M. Vázquez y E. Mansur, pp. 541-558. Editorial Utopías, Ushuaia.

Stern, C.R. 2018. Obsidian sources and distribution in Patagonia, southernmost South America. Quaternary International 468:190-205.

Strauss, L.G. 1991. Underground archaeology: Perspectives on caves and rockshelters. En Archaeological Method and Theory 2, editado por M.B. Schiffer y C.L Costin, pp. 255-304. The University of Arizona Press, Tucson.

Stuiver, M., P.J. Reimer y R.W. Reimer 2018. CALIB 7.4 [WWW program].

Thompson, B. y C. Méndez 2019. Altos del Moro 2: una ocupación a cielo abierto ca. 2000 años cal A.P. en los bosques occidentales de Patagonia Central. Actas del XX Congreso Nacional de Arqueología Chilena, pp. 249-260. Sociedad Chilena de Arqueología y Universidad de Concepción, Concepción.

Thorndycraft, V.R., J.M. Bendle, G. Benito, B.J. Davies, C. Sancho, A.P. Palmer, D. Fabel, A. Medialdea y J.R.V. Martin 2019. Glacial lake evolution and Atlantic-Pacific drainage reversals during deglaciation of the Patagonian Ice Sheet. Quaternary Science Reviews 203:102-127.

Velásquez, H., C. Méndez, O. Reyes, V. Trejo, L. Sanhueza, D. Quiroz y D. Jackson 2007. Campamentos residenciales tardíos a cielo abierto en el alto rio Cisnes (XI Región de Aisén): Appeleg 1 (CIS 009). Magallania 35 (1):85-98.

Veth, P 1993. Islands in the Interior: The Dynamics of Prehistoric Adaptations within the Arid Zone of Australia. International Monographs in Prehistory, Ann Arbor.

Villa-Martínez, R., P.I. Moreno y M.A. Valenzuela 2012. Deglacial and postglacial vegetation changes on the eastern slopes of the central Patagonian Andes ( $\left.47^{\circ} \mathrm{S}\right)$. Quaternary Science Reviews 32:86-99.

Ward, G.K., y S.R. Wilson 1978. Procedures for comparing and combining radiocarbon age determinations: A critique. Archaeometry 20 (1):19-31.

\section{Nota}

1 Una muestra ósea del perfil B resultó en una edad de $930 \pm 30$ AP (UGAMS 39295) con un valor de C/N=9.68, por lo que fue considerada no confiable y excluida de la muestra. 
